\title{
Uncertainties and assessments of chemistry-climate models of the stratosphere
}

\author{
J. Austin ${ }^{1}$, D. Shindell ${ }^{2}$, S. R. Beagley ${ }^{3}$, C. Brühl ${ }^{4}$, M. Dameris ${ }^{5}$, E. Manzini ${ }^{6}$, T. Nagashima ${ }^{7}$, P. Newman ${ }^{8}$, \\ S. Pawson ${ }^{8}$, G. Pitari ${ }^{9}$, E. Rozanov ${ }^{10}$, C. Schnadt ${ }^{5}$, and T. G. Shepherd ${ }^{11}$ \\ ${ }^{1}$ Meteorological Office, London Rd., Bracknell, Berks., RG12 2SZ, UK \\ ${ }^{2}$ NASA-Goddard Institute for Space Studies, 2880 Broadway, New York, NY 10025, USA \\ ${ }^{3}$ York University, Canada \\ ${ }^{4}$ Max Planck Institut für Chemie, Mainz, Germany \\ ${ }^{5}$ DLR, Oberpfaffenhofen, Weßling, Germany \\ ${ }^{6}$ Max Planck Institut für Meteorologie, Hamburg, Germany \\ ${ }^{7}$ Center for Climate System Research, University of Tokyo, Japan \\ ${ }^{8}$ Goddard Earth Sciences and Technology Center, NASA/Goddard Space Flight Center Code 916, Greenbelt, MD 20771, \\ USA \\ ${ }^{9}$ Dipartamento di Fisica, Università de L'Aquila, 67010 Coppito, L'Aquila, Italy \\ ${ }^{10}$ PMOD-WRC/ IAC ETH, Dorfstrasse 33, Davos Dorf CH-7260, Switzerland \\ ${ }^{11}$ Department of Physics, University of Toronto, Toronto, Ontario, Canada
}

Received: 28 May 2002 - Published in Atmos. Chem. Phys. Discuss.: 12 July 2002

Revised: 9 September 2002 - Accepted: 24 September 2002 - Published: 9 January 2003

\begin{abstract}
In recent years a number of chemistry-climate models have been developed with an emphasis on the stratosphere. Such models cover a wide range of time scales of integration and vary considerably in complexity. The results of specific diagnostics are here analysed to examine the differences amongst individual models and observations, to assess the consistency of model predictions, with a particular focus on polar ozone. For example, many models indicate a significant cold bias in high latitudes, the "cold pole problem", particularly in the southern hemisphere during winter and spring. This is related to wave propagation from the troposphere which can be improved by improving model horizontal resolution and with the use of non-orographic gravity wave drag. As a result of the widely differing modelled polar temperatures, different amounts of polar stratospheric clouds are simulated which in turn result in varying ozone values in the models.

The results are also compared to determine the possible future behaviour of ozone, with an emphasis on the polar regions and mid-latitudes. All models predict eventual ozone recovery, but give a range of results concerning its timing and extent. Differences in the simulation of gravity waves and planetary waves as well as model resolution are likely major sources of uncertainty for this issue. In the Antarctic, the ozone hole has probably reached almost its deepest although
\end{abstract}

Correspondence to: J. Austin (john.austin@metoffice.com) the vertical and horizontal extent of depletion may increase slightly further over the next few years. According to the model results, Antarctic ozone recovery could begin any year within the range 2001 to 2008 .

The limited number of models which have been integrated sufficiently far indicate that full recovery of ozone to 1980 levels may not occur in the Antarctic until about the year 2050. For the Arctic, most models indicate that small ozone losses may continue for a few more years and that recovery could begin any year within the range 2004 to 2019 . The start of ozone recovery in the Arctic is therefore expected to appear later than in the Antarctic.

Further, interannual variability will tend to mask the signal for longer than in the Antarctic, delaying still further the date at which ozone recovery may be said to have started. Because of this inherent variability of the system, the decadal evolution of Arctic ozone will not necessarily be a direct response to external forcing.

\section{Introduction}

The extent to which stratospheric change can influence climate is only beginning to be understood. Before the discovery of the Antarctic ozone hole (Farman et al., 1985) it was thought that increases in the concentration of $\mathrm{CO}_{2}$ 
would cool the stratosphere and increase ozone (e.g. Groves and Tuck, 1980). More generally it is now recognised that increases in the other well-mixed greenhouse gases (WMGHGs), $\mathrm{CH}_{4}, \mathrm{~N}_{2} \mathrm{O}$ and chlorofluorocarbons, also cool the stratosphere slightly. In polar regions, an increase in WMGHGs may increase Polar Stratospheric Clouds (PSCs) and decrease ozone in the Arctic (Austin et al., 1992) via the same processes which produce the Antarctic ozone hole (Solomon, 1986). Changes to the thermal structure of the stratosphere also affect the wind fields which determine the transport of ozone and the long-lived species that chemically control ozone. Decreases in stratospheric ozone change tropospheric chemistry by increasing the amount of UV radiation reaching the troposphere, and also cool the troposphere as ozone is itself a greenhouse gas. Consequently, atmospheric chemistry and climate are coupled in important ways. However, the degree of coupling is unclear. For example, Austin et al. (1992) showed that a doubling of $\mathrm{CO}_{2}$ concentrations, expected towards the end of the 21 st century, could lead to severe Arctic ozone loss if large halogen abundances persisted until that time.

On the other hand, the calculations of Pitari et al. (1992) showed only a slight reduction in Arctic ozone due to a $\mathrm{CO}_{2}$ doubling, while again keeping halogen amounts fixed.

Since the early 1990s, the amendments to the Montreal Protocol have resulted in a considerable constraint on the evolution of halogen amounts and more recent calculations have been able to take this into consideration. For example, in a coupled chemistry-climate simulation, Shindell et al. (1998a) calculated much increased ozone depletion over the next decade or so, with severe ozone loss in the Arctic in some years. In contrast, recent results from other models (Austin et al., 2000; Brühl et al., 2001; Nagashima et al., 2002; Schnadt et al., 2002) indicate a relatively small change in Arctic ozone over the next few decades. The net effect on ozone and temperature is that due to both the increased radiative cooling from WMGHG increases and due to changes in downwelling (and adiabatic warming) from planetary wave drag. The latter changes could decrease ozone if planetary wave activity decreases, thus enhancing the radiative signal; or if planetary wave activity increases, the radiative signal could be damped or even reversed causing an increase in ozone. This illustrates one of the problems concerning future predictions: the simulation of the north polar stratospheric temperature, and thus ozone, is complicated by the models' ability to reproduce realistically the dynamical activity of this region. The model results therefore crucially depend on the length of the numerical simulation and model configuration, such as spatial resolution, position of the model upper boundary, gravity wave drag (gwd) scheme and the degree of coupling between the chemically active species and the radiation scheme. The fact that the results are model dependent emphasises that the various dynamical-chemical feedback mechanisms in the atmosphere, as well as the dynamical coupling between troposphere and stratosphere, are not yet fully understood.

These mechanisms are here explored by comparing specific diagnostics from a range of coupled chemistry-climate models. The primary interest is polar ozone and factors that control it, such as PSCs. Via transport, polar ozone also has an impact on middle latitudes which are also considered briefly. In Sect. 2, the models are described and in Sect. 3, model diagnostics are compared amongst the models and with observations. Having established model strengths and weaknesses, their results are assessed in Sect. 4, to try to provide a consensus on the likely trend in future ozone. Conclusions are drawn in Sect. 5.

\section{Models used in the comparisons}

Climate models have in the past been run with fixed WMGHGs for both present and doubled $\mathrm{CO}_{2}$ with the investigation of the subsequent "equilibrium climate". Several coupled chemistry-climate runs have followed this route with multi-year "time slice" simulations applicable to WMGHG concentrations for specific years (e.g. Rozanov et al., 2001; Schnadt et al., 2002; Pitari et al., 2002; Steil et al., 2002). Other climate simulations have involved transient changes in the WMGHGs, and several coupled chemistry-climate simulations have followed this pattern (Shindell et al., 1998a; Austin, 2002; Nagashima et al., 2002). The advantage of transient experiments is that the detailed evolution of ozone can be determined in the same way that it is likely to occur (in principle) in the atmosphere, albeit with some statistical error. The impact of interannual variability can be assessed by determining this variability over, e.g. a 10-year period. Comparisons with observations are more direct since the concentrations of the halogens and WMGHGs are changing to the same extent in the model as in the atmosphere. Time slice simulations need a sufficient duration (at least 10 and preferably 20 years) to allow the interannual variability to be determined, but in principle, 20-year evaluations of the same conditions may have less variability than the same period in the atmosphere in which halogens may be changing rapidly. Time slice runs also have the advantage that several realisations of the same year are available, from which future predictions can be assessed. However, in practice it may be better to examine the behaviour of different models, since a given model will tend to have systematic errors. Both transient simulations and time slice simulations are here used, to bring together the best of both sets of simulations.

The models used in the comparisons are indicated in Table 1 , in order of decreasing horizontal resolution. All the models have a comprehensive range of chemical reactions except that in the GISS model the chemistry is parameterised. $U L A Q$ is the only model with a substantial aerosol package but has diurnally averaged chemistry. This model has been run in time slice mode. Of the other models run in this mode, CMAM and MAECHAM/CHEM have a high upper bound- 
Table 1. Models used in the comparisons. All the models use scenario IS92a of IPCC (1992) for the WMGHGs and WMO (1999), Chapter 12, for the halogen amounts except the CMAM, ULAQ and CCSR/NIES models. For the WMGHGs, CMAM uses observations for 1987 and the same values for 2000; the 2028 run uses WMGHGs appropriate to 2045 according to IS92a. ULAQ and CCSR/NIES use WMO (1999) for both the halogens and the WMGHGs. For the transient runs the length of the runs is indicated by a range of years. For the time slice runs, the date is given first, and the length is given second. Thus $1980 \times 15$ is understood to be 1980 conditions for 15 years. Dates and times include any spinup period

\begin{tabular}{|c|c|c|c|c|}
\hline Name & $\begin{array}{l}\text { Horizontal } \\
\text { Resolution }\end{array}$ & $\begin{array}{l}\text { No. of Levels/ } \\
\text { Upper boundary }\end{array}$ & $\begin{array}{l}\text { Length of } \\
\text { Simulations }\end{array}$ & References \\
\hline UMETRAC & $2.5^{\circ} \times 3.75^{\circ}$ & $64 / 0.01 \mathrm{hPa}$ & $\begin{array}{l}1979-2020 \\
1975-2020\end{array}$ & $\begin{array}{c}\text { Austin (2002), } \\
\text { Austin and Butchart (2002) }\end{array}$ \\
\hline CMAM & $\mathrm{T} 32$ & $65 / 0.0006 \mathrm{hPa}$ & $\begin{array}{c}1987,2000 \times 12 \\
2028 \times 12\end{array}$ & de Grandpré et al. (2000) \\
\hline $\begin{array}{l}\text { MAECHAM/ } \\
\text { CHEM }\end{array}$ & $\mathrm{T} 30$ & $39 / 0.01 \mathrm{hPa}$ & $\begin{array}{l}1960,1990 \times 22 \\
2000,2030 \times 22\end{array}$ & $\begin{array}{l}\text { Steil et al. (2002), } \\
\text { Manzini et al. (2002) }\end{array}$ \\
\hline $\mathrm{E} 39 / \mathrm{C}$ & $\mathrm{T} 30$ & $39 / 10 \mathrm{hPa}$ & $\begin{array}{l}1960,1980 \times 22 \\
1990,2015 \times 22\end{array}$ & $\begin{array}{l}\text { Schnadt et al. (2002) } \\
\text { Hein et al. (2001) }\end{array}$ \\
\hline UIUC & $4^{\circ} \times 5^{\circ}$ & $25 / 1 \mathrm{hPa}$ & $1995 \times 15$ & Rozanov et al. (2001) \\
\hline CCSR/NIES & $\mathrm{T} 21$ & $34 / 0.01 \mathrm{hPa}$ & $1986-2050$ & $\begin{array}{l}\text { Takigawa et al. (1999), } \\
\text { Nagashima et al. (2002) }\end{array}$ \\
\hline GISS & $8^{\circ} \times 10^{\circ}$ & $23 / 0.002 \mathrm{hPa}$ & 1959-2067 & Shindell et al. (1998a) \\
\hline ULAQ & $10^{\circ} \times 20^{\circ}$ & $26 / 0.04 \mathrm{hPa}$ & $\begin{array}{c}1990 \times 20 \\
2030 \times 5\end{array}$ & Pitari et al. (2002) \\
\hline
\end{tabular}

ary $(0.01 \mathrm{hPa}$ and above), while UIUC and $E 39 / C$ have a much lower upper boundary (1 hPa and below). UMETRAC, CCSR/NIES and GISS have been run in transient mode, although the extra cost of the UMETRAC model has reduced the total integration time compared with the other two models. In Sects. 3 and 4, model diagnostics are presented for each model for all available quantities, but these differ between models depending on archival procedures. For example, only monthly mean data were saved for the GISS runs, so quantities derived from daily data, such as heat fluxes or PSC frequencies, are not shown. Brief summaries of the models follow.

\subsection{Model descriptions}

\section{UMETRAC}

The Unified Model with Eulerian TRansport And Chemistry is based on the Meteological Office's Unified Model (UM), which has been used in the Intergovernmental Panel on Climate Change (IPCC) assessments (e.g. IPCC, 2001). UMETRAC has 64 vertical levels, from the ground to $0.01 \mathrm{hPa}$, and includes a coupled stratospheric chemistry scheme involving 13 advected tracers $\left(\mathrm{O}_{3}, \mathrm{HNO}_{3}, \mathrm{~N}_{2} \mathrm{O}_{5}, \mathrm{HNO}_{4}\right.$, $\mathrm{HCl}(\mathrm{g}+\mathrm{s}), \mathrm{HOCl}, \mathrm{ClONO}_{2}, \mathrm{HOBr}, \mathrm{HBr}, \mathrm{BrONO}_{2}, \mathrm{H}_{2} \mathrm{O}_{2}$, $\mathrm{H}_{2} \mathrm{CO}$, Tracer-1). Tracer-1 is used to parameterise the longlived species and families $\mathrm{H}_{2} \mathrm{O}, \mathrm{CH}_{4}, \mathrm{Cl}_{\mathrm{y}}, \mathrm{Br}_{\mathrm{y}}, \mathrm{H}_{2} \mathrm{SO}_{4}$ and $\mathrm{NO}_{\mathrm{y}}$, where $\mathrm{Cl}_{\mathrm{y}}=\mathrm{Cl}+\mathrm{ClO}+\mathrm{HCl}(\mathrm{g}+\mathrm{s})+\mathrm{HOCl}+\mathrm{ClONO}_{2}$ $+2 \mathrm{Cl}_{2} \mathrm{O}_{2}+\mathrm{BrCl}, \mathrm{Br}_{\mathrm{y}}=\mathrm{Br}+\mathrm{BrO}+\mathrm{HBr}+\mathrm{BrONO}_{2}+$ $\mathrm{HOBr}+\mathrm{BrCl}$, and $\mathrm{NO}_{\mathrm{y}}=\mathrm{NO}+\mathrm{NO}_{2}+\mathrm{NO}_{3}+\mathrm{HNO}_{3}(\mathrm{~g}+\mathrm{s})$
$+2 \mathrm{~N}_{2} \mathrm{O}_{5}+\mathrm{HNO}_{4}+\mathrm{ClONO}_{2}+\mathrm{BrONO}_{2}$. In the above $(\mathrm{g}+\mathrm{s})$ is used to denote the sum of concentrations in the gas $(\mathrm{g})$ and solid (s) phases. A simplified sedimentation scheme is included and the PSC scheme is based on liquid ternary solutions (LTS) and ice or nitric acid trihydrate (NAT) and water ice. The photochemistry therefore explicitly includes all the major processes affecting stratospheric ozone. Reaction rates are taken from DeMore et al. (1997) and Sander et al. (2000). Sea surface temperatures and sea ice are specified from observations or from simulations of a coupled oceanatmosphere version of the model. The dynamics is available using Rayleigh friction as standard to decelerate the jet or with a non-orographic gwd scheme. Halogen amounts are specified according to WMO (1999), Tables 1-2, and the greenhouse gas concentrations $\left(\mathrm{CO}_{2}, \mathrm{CH}_{4}\right.$ and $\left.\mathrm{N}_{2} \mathrm{O}\right)$ are specified according to IPCC scenario IS92a (IPCC, 1992). Simulations have been completed for the periods 1979-2020 (Rayleigh friction) and 1975-2020 (non-orographic gwd). The results of the model for the Rayleigh friction and nonorographic gwd calculations are described in Austin (2002) and Austin and Butchart (2002).

\section{CMAM}

The Canadian Middle Atmosphere Model is an extension of the Canadian Centre for Climate Modelling and Analysis (CCCma) spectral General Circulation Model (GCM) up to $0.0006 \mathrm{hPa}$, roughly $100 \mathrm{~km}$ altitude (Beagley et al., 1997). The version used here has a T32 spectral truncation and 65 vertical levels. The model includes a full representa- 
tion of stratospheric chemistry with all the relevant catalytic ozone loss cycles (de Grandpré et al., 1997). There are 31 non-advected species and 16 advected species and families $\mathrm{HNO}_{4}, \mathrm{HBr}, \mathrm{CO}, \mathrm{H}_{2}, \mathrm{CH}_{3} \mathrm{Br}, \mathrm{CH}_{4}, \mathrm{~N}_{2} \mathrm{O}, \mathrm{H}_{2} \mathrm{O}, \mathrm{CFC}-11$, $\mathrm{CFC}-12, \mathrm{O}_{\mathrm{x}}\left(=\mathrm{O}\left({ }^{1} \mathrm{D}\right)+\mathrm{O}\left({ }^{3} \mathrm{P}\right)+\mathrm{O}_{3}\right), \mathrm{NO}_{\mathrm{x}}\left(=\mathrm{NO}+\mathrm{NO}_{2}\right.$ $\left.+\mathrm{NO}_{3}+2 \mathrm{~N}_{2} \mathrm{O}_{5}\right), \mathrm{ClO}_{\mathrm{x}}(=\mathrm{ClO}+\mathrm{OClO}+\mathrm{HOCl}+\mathrm{Cl}+$ $\left.\mathrm{ClONO}_{2}+2 \mathrm{Cl}_{2} \mathrm{O}_{2}+2 \mathrm{Cl}_{2}+\mathrm{HCl}(\mathrm{g}+\mathrm{s})\right), \mathrm{BrO}_{\mathrm{x}}(=\mathrm{Br}+\mathrm{BrO}+$ $\left.\mathrm{BrCl}+\mathrm{BrONO}_{2}+\mathrm{HOBr}\right), \mathrm{HO}_{\mathrm{x}}\left(=\mathrm{OH}+\mathrm{HO}_{2}+\mathrm{H}+2 \mathrm{H}_{2} \mathrm{O}_{2}\right)$, and $\mathrm{HNO}_{3}(\mathrm{~g}+\mathrm{s})$. Chemical transport is accomplished using spectral advection. Heterogeneous reactions are included for sulphate aerosols, LTS, and water ice, without sedimentation.

There is currently no parameterization of NAT PSCs, or of any associated denitrification. PSC threshold temperatures are not adjusted to compensate for temperature biases. Chemical reaction rates follow DeMore et al. (1997) and Sander et al. (2000). The chemistry is fully interactive with the radiation code (de Grandpré et al., 2000). For these runs, gravity wave drag is parameterised with the McFarlane orographic scheme and the Hines non-orographic scheme (McLandress, 1998; Beagley et al., 2000).

The time-slice experiments are performed for 12 years, with the first two years discarded. Halogens are specified from WMO (1999), Chapter 12, and are identical in the 1987 and 2028 runs. For the 1987 and 2000 runs, WMGHGs $\left(\mathrm{CO}_{2}, \mathrm{CH}_{4}\right.$ and $\left.\mathrm{N}_{2} \mathrm{O}\right)$ are specified from observations as of 1987 , and the sea surface temperatures and sea-ice distributions are taken from Shea et al. (1990). For the 2028 run, WMGHGs are taken from the IPCC IS92a scenario while sea-surface temperatures and sea-ice distributions are altered according to the anomalies predicted from a transient simulation with the CCCma coupled climate model (Boer et al., 2000); however due to an error in the specification of the experiments, these forcings are appropriate to 2045 rather than 2028.

\section{MAECHAM/CHEM}

This is a coupled chemistry-climate model based on the MAECHAM4 climate model. The chemistry module (Steil et al., 1998) adopts the "family" technique allowing time steps to be as large as $45 \mathrm{~min}$ and includes reactive chlorine, nitrogen and hydrogen, and methane oxidation. Photolysis rates are calculated on-line using the fast and accurate scheme of Landgraf and Crutzen (1998) to account for varying clouds and overhead ozone.

Heterogeneous reactions on NAT and ice PSCs, and on sulphate aerosol are included, as well as sedimentation of ice PSC particles. Since it is assumed that ice grows on NAT particles, this accounts to some extent for denitrification if it is cold enough for ice. NAT PSCs are calculated from the model temperature, $\mathrm{H}_{2} \mathrm{O}$ and $\mathrm{HNO}_{3}$ using the formulae of Hanson and Mauersberger (1988), without assuming a nucleation barrier arguing that subgrid scale temperature fluctuations produce the required supercooling. The gravity wave parameterization consists of two parts, separately representing momentum flux deposition due to orographic gravity waves and a broad band spectrum of non-orographic gravity waves (Manzini and McFarlane, 1998). A modified version of the McFarlane (1987) parameterization is used to account for the orographic gwd.

Chemical species are advected using the fluxform semiLagrangian transport scheme of Rasch and Lawrence (1998). For the time slice experiments the model is integrated about 22 years (including spinup) using fixed surface mixing ratios of source gases (WMO, 1999) and ten years average seasonal sea surface temperture distributions based on Hadley Centre data. For the simulations of future conditions, the average sea surface temperatures have been taken from the transient climate simulation of Roeckner et al (1999).

\section{E39/C}

A detailed description of this chemistry-climate model, also known as ECHAM4.L39(DLR)/CHEM, has been given by Hein et al. (2001), who also discussed the main features of the model climatology. The model horizontal resolution is T30 with a corresponding Gaussian transform latitudelongitude grid of mesh size $3.75^{\circ} \times 3.75^{\circ}$ on which model physics, chemistry, and tracer transport are calculated. The model has 39 layers from the surface to the top layer centred at $10 \mathrm{hPa}$ (Land et al., 1999) and has particularly high resolution near the tropopause. A parameterization for orographic gwd (Miller et al., 1989) is employed, but the effects of non-orographic gravity waves are not considered. The chemistry module CHEM (Steil et al., 1998, updated Hein et al., 2001) uses reaction rate coefficients from DeMore et al. (1997) and is based on the family concept, containing the most relevant chemical compounds and reactions necessary to simulate upper tropospheric and lower stratospheric ozone chemistry, including heterogeneous chemical reactions on PSCs and sulphate aerosol, as well as tropospheric $\mathrm{NO}_{\mathrm{x}}-\mathrm{HO}_{\mathrm{x}}-\mathrm{CO}-\mathrm{CH}_{4}-\mathrm{O}_{3}$ chemistry. The advected tracers are $\mathrm{CH}_{4}, \mathrm{HCl}, \mathrm{H}_{2} \mathrm{O}_{2}, \mathrm{CO}, \mathrm{CH}_{3} \mathrm{O}_{2} \mathrm{H}, \mathrm{ClONO}_{2}$, $\mathrm{O}_{\mathrm{x}}\left(=\mathrm{O}\left({ }^{1} \mathrm{D}\right)+\mathrm{O}\left({ }^{3} \mathrm{P}\right)+\mathrm{O}_{3}\right), \mathrm{NO}_{\mathrm{x}}\left(=\mathrm{N}+\mathrm{NO}+\mathrm{NO}_{2}+\mathrm{NO}_{3}\right.$ $\left.+2 \mathrm{~N}_{2} \mathrm{O}_{5}+\mathrm{HNO}_{4}\right), \mathrm{ClO}_{\mathrm{x}}(=\mathrm{Cl}+\mathrm{ClO}+\mathrm{HOCl}), \mathrm{HNO}_{3}$ + NAT and $\mathrm{H}_{2} \mathrm{O}+$ ICE. Physical, chemical, and transport processes are calculated simultaneously at each time step, which is fixed to $30 \mathrm{~min}$. The PSCs in the model are based on NAT and ice amounts depending on the temperature and the concentration of $\mathrm{HNO}_{3}$ and $\mathrm{H}_{2} \mathrm{O}$ (Hanson and Mauersberger, 1988). A nucleation barrier is prescribed to account for the observed super-saturation of NAT-particles (Schlager et al., 1990; Peter et al., 1991; Dye et al., 1992). A simplified scheme for the sedimentation of NAT and ice particles is applied. Stratospheric sulphuric acid aerosol surface areas are based on background conditions (WMO, 1992, Chapter 3 ) with a coarse zonal average. Sea surface temperature and sea ice distributions are prescribed for the various time 
slices according to the greenhouse gas driven transient climate change simulations of Roeckner et al. (1999).

\section{UIUC}

The University of Illinois at Urbana-Champaign model is a grid-point GCM with interactive chemistry (Yang et al., 2000; Rozanov et al., 2001). The model horizontal resolution is $4^{\circ}$ latitude and $5^{\circ}$ longitude, with 24 layers spanning the atmosphere from the surface to $1 \mathrm{hPa}$. The chemicaltransport part of the model simulates the time-dependent three-dimensional distributions of 42 chemical species $\left(\mathrm{O}_{3}\right.$, $\mathrm{O}\left({ }^{1} \mathrm{D}\right), \mathrm{O}\left({ }^{3} \mathrm{P}\right), \mathrm{N}, \mathrm{NO}, \mathrm{NO}_{2}, \mathrm{NO}_{3}, \mathrm{~N}_{2} \mathrm{O}_{5}, \mathrm{HNO}_{3}, \mathrm{HNO}_{4}$, $\mathrm{N}_{2} \mathrm{O}, \mathrm{H}, \mathrm{OH}, \mathrm{HO}_{2}, \mathrm{H}_{2} \mathrm{O}_{2}, \mathrm{H}_{2} \mathrm{O}, \mathrm{H}_{2}, \mathrm{Cl}, \mathrm{ClO}, \mathrm{HCl}, \mathrm{HOCl}$, $\mathrm{ClONO}_{2}, \mathrm{Cl}_{2}, \mathrm{Cl}_{2} \mathrm{O}_{2}, \mathrm{CF}_{2} \mathrm{Cl}_{2}, \mathrm{CFCl}_{3}, \mathrm{Br}, \mathrm{BrO}, \mathrm{BrONO}_{2}$, $\mathrm{HOBr}, \mathrm{HBr}, \mathrm{BrCl}, \mathrm{CBrF}_{3}, \mathrm{CH}_{3} \mathrm{Br}, \mathrm{CO}, \mathrm{CH}_{4}, \mathrm{CH}_{3}, \mathrm{CH}_{3} \mathrm{O}_{2}$, $\mathrm{CH}_{3} \mathrm{OOH}, \mathrm{CH}_{3} \mathrm{O}, \mathrm{CH}_{2} \mathrm{O}$, and $\mathrm{CHO}$ ), which are determined by 199 gas-phase and photolysis reactions. The model also takes into account 6 heterogeneous reactions on and in sulphate aerosol and polar stratospheric cloud particles. The chemical solver is based on the pure implicit iterative Newton-Raphson scheme (Rozanov et al., 1999). The reaction coefficients are taken from DeMore et al. (1997) and Sander et al. (2000).

Photolysis rates are calculated at every chemical step using a look-up-table (Rozanov et al., 1999). The advective transport of species is calculated using the hybrid advection scheme proposed by Zubov et al. (1999). A simplified scheme is applied for the calculation of the PSC particles (NAT and ice) formation and sedimentation. Sea surface temperature and sea ice distributions are prescribed from the AMIP-II monthly mean distributions, which are the averages from 1979 through 1996 (Gleckler, 1996). The orographic scheme of Palmer et al. (1986) is used to parameterise gravity wave drag.

In this paper the results of a 15-year long control run of the model will be considered. The climatology of this run has been presented by Rozanov et al. (2001).

\section{CCSR/NIES}

An overview of the Center for Climate System Research/National Institute for Environmental Studies GCM with an interactive stratospheric chemistry module has been described in Takigawa et al. (1999), and some updates for the present integration are given by Nagashima et al. (2002). The horizontal resolution is $\mathrm{T} 21$ (longitude $\times$ latitude $\simeq 5.6^{\circ} \times$ $5.6^{\circ}$ ) and the vertical domain extends from the surface to about $0.01 \mathrm{hPa}$ with 34 layers. Orographic gwd is parameterised following McFarlane (1987), but non-orographic gwd is not included. In the model, 19 species and 5 chemical families are explicitly transported: $\mathrm{CH}_{4}, \mathrm{~N}_{2} \mathrm{O}, \mathrm{H}_{2} \mathrm{O}_{2}, \mathrm{H}_{2} \mathrm{O}(\mathrm{g}+\mathrm{s})$, $\mathrm{HNO}_{3}+\mathrm{NAT}, \mathrm{HNO}_{4}, \mathrm{~N}_{2} \mathrm{O}_{5}, \mathrm{ClONO}_{2}, \mathrm{HCl}, \mathrm{HOCl}, \mathrm{Cl}_{2}$, $\mathrm{ClNO}_{2}, \mathrm{CCl}_{4}, \mathrm{CFC11}, \mathrm{CFC12}, \mathrm{CFC113}, \mathrm{HCFC} 22, \mathrm{CH}_{3} \mathrm{Cl}$, $\mathrm{CH}_{3} \mathrm{CCl}_{3}, \mathrm{O}_{\mathrm{x}}, \mathrm{HO}_{\mathrm{x}}\left(=\mathrm{H}+\mathrm{OH}+\mathrm{HO}_{2}\right), \mathrm{NO}_{\mathrm{x}}(=\mathrm{N}+\mathrm{NO}$
$\left.+\mathrm{NO}_{2}+\mathrm{NO}_{3}\right), \mathrm{ClO}_{\mathrm{x}}\left(=\mathrm{Cl}+\mathrm{ClO}+\mathrm{ClOO}+\mathrm{Cl}_{2} \mathrm{O}_{2}\right), \mathrm{NO}_{\mathrm{y}}$ and $\mathrm{Cl}_{\mathrm{y}}$. The amount of NAT and ice PSCs are calculated using the relationship of Hanson and Mauersberger (1988) and of Murray (1967), respectively. As in E39/C, a nucleation barrier is also assumed.

Reaction rate coefficients are taken primarily from DeMore et al. (1997) and Sander et al. (2000). A 65-year transient integration was performed for the period 19862050 including the trends of chlorine-containing substances (WMO, 1999, Table 12-2) and those of greenhouse gases (Also WMO, 1999, Table 12-2, very similar to IPCC scenario IS92a, IPCC, 1992). The expected sea surface temperature variation, separately calculated by the CCSR/NIES coupled ocean-atmospheric GCM, is also applied.

\section{GISS}

The model used here is a version of the Goddard Institute for Space Studies stratospheric climate model which has $8^{\circ} \times 10^{\circ}$ horizontal resolution, and 23 vertical layers extending up into the mesosphere (top at $0.002 \mathrm{hPa}, \sim 85 \mathrm{~km}$ ) (Rind et al., 1988a, b). This atmospheric model is coupled to a mixed-layer ocean, allowing sea surface temperatures to respond to external forcings. A gravity wave parameterization is employed in the model in which the temperature and wind fields in each grid box are used to calculate gravity wave effects due to wind shear, convection, and topography. For the runs described here, the mountain drag was set to one quarter the original value which gives a better reproduction of observed temperatures in the polar lower stratosphere of each hemisphere during their respective winter and spring periods. The model includes a simple parameterization of the heterogeneous chemistry responsible for polar ozone depletion (Shindell et al., 1998a). Chlorine activation takes place whenever temperatures fall below $195 \mathrm{~K}$. Ozone depletion is then calculated at each location with active chlorine and sunlight using the reaction rates of DeMore et al. (1997). An additional contribution of $15 \%$ from bromine chemistry is also included. When heterogeneous processing ceases, chlorine deactivation follows two-dimensional model-derived rates (Shindell et al., 1998b). Ozone recovery rates are parameterised so that ozone losses are restored based on the photochemical lifetime of ozone at each altitude. Changes in ozone transport are not included interactively, but are calculated after the model runs and included in the results shown here. The model was run with increasing greenhouse gas concentrations starting in 1959 and continuing until 2067. The trends in greenhouse gas concentrations were based on observations until 1985, and subsequently followed the IPCC projections IS92a (IPCC, 1992), although the observed rates of increase since 1985 have been somewhat less. 

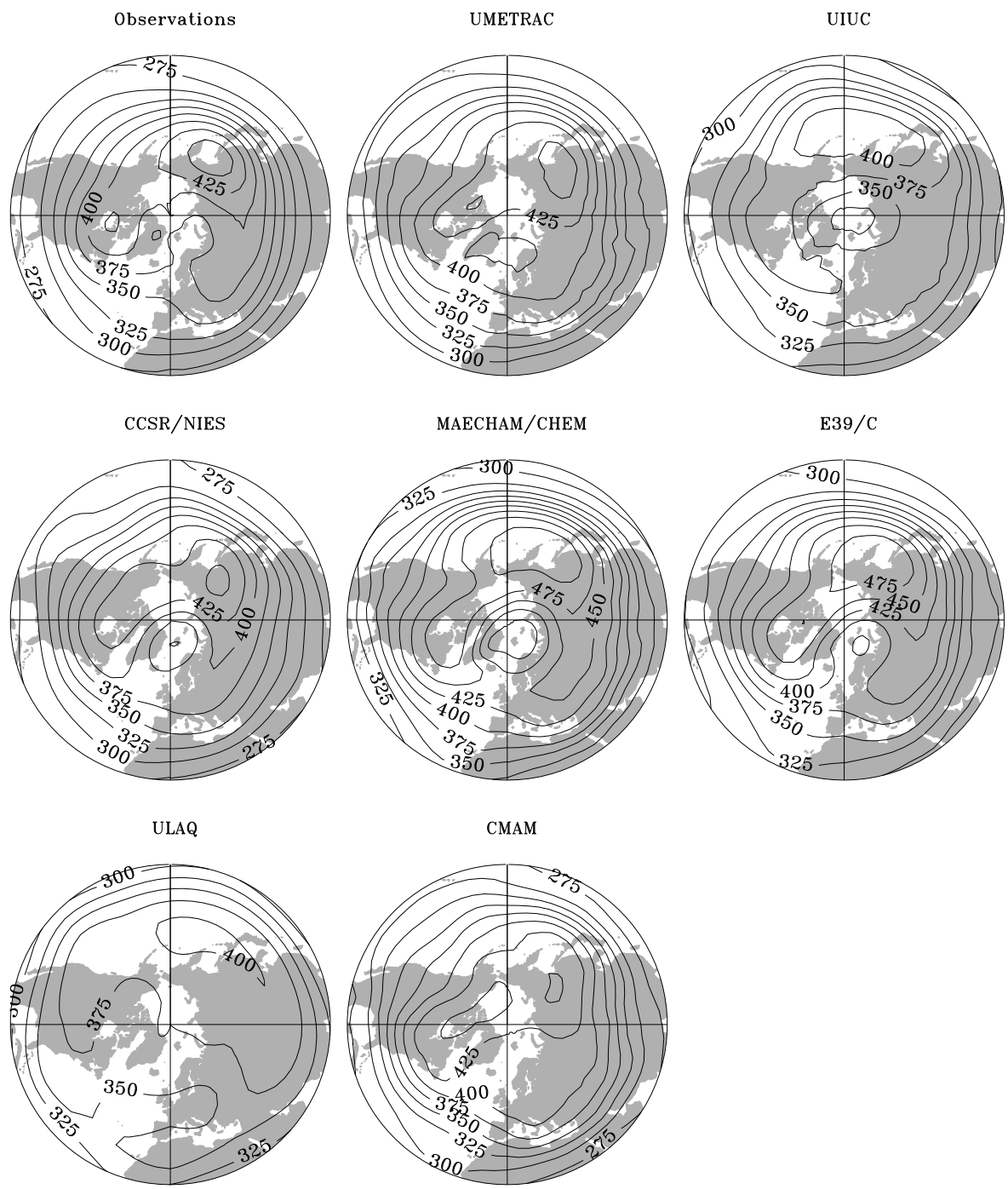

Fig. 1. Monthly mean total ozone (DU) over the northern hemisphere for the month of March for the current atmosphere for participating models. The observations are taken from a range of satellite data (see text) for the period 1993-2000. The model climatologies are as follows: UMETRAC: mean 1980-2000, CMAM: 2000 run, MAECHAM/CHEM: 2000 run, E39/C: 1990 run, CCSR/NIES: mean 1990-1999, UIUC: 1995 run, ULAQ, 1990 run.

\section{$U L A Q$}

The University of L'Aquila model is a low-resolution coupled chemistry-climate model, which has been extensively used in the IPCC assessment (IPCC, 2001). The chemicaltransport module has a resolution of $10^{\circ} \times 20^{\circ}$ in latitudelongitude. There are 26 log-pressure levels, from the ground to about $0.04 \mathrm{hPa}$, giving an approximate vertical resolution of $2.8 \mathrm{~km}$. Dynamical fields (streamfunction, velocity potential and temperature) are taken from the output of a spectral GCM (Pitari et al., 2002). Vertical diffusion is used to simulate those processes not explicitly included in the model such as the effect of breaking gravity waves. See Pitari et al. (1992) and Pitari et al. (2002) for further details of the model.
The Chemical Transport Model (CTM) and GCM are coupled via the dynamical fields and via the radiatively active species $\left(\mathrm{H}_{2} \mathrm{O}, \mathrm{CH}_{4}, \mathrm{~N}_{2} \mathrm{O}, \mathrm{CFCs}, \mathrm{O}_{3}, \mathrm{NO}_{2}\right.$, and aerosols). All chemical species are diurnally averaged. The medium and short-lived chemical species are grouped into families: $\mathrm{O}_{\mathrm{x}}, \mathrm{NO}_{\mathrm{x}}, \mathrm{NO}_{\mathrm{y}}\left(=\mathrm{NO}_{\mathrm{x}}+\mathrm{HNO}_{3}\right), \mathrm{HO}_{\mathrm{x}}, \mathrm{CHO}_{\mathrm{x}}, \mathrm{Cl}_{\mathrm{y}}, \mathrm{Br}_{\mathrm{y}}$, $\mathrm{SO}_{\mathrm{x}}$ and aerosols. The long-lived and surface-flux species in the model are $\mathrm{N}_{2} \mathrm{O}, \mathrm{CH}_{4}, \mathrm{H}_{2} \mathrm{O}, \mathrm{CO}$, NMHC, CFCs, HCFCs, halons, OCS, $\mathrm{CS}_{2}$, DMS, $\mathrm{H}_{2} \mathrm{~S}, \mathrm{SO}_{2}$ for a total of 40 transported species (plus 57 aerosol size categories) as well as 26 species at photochemical equilibrium. All photochemical data are taken from DeMore et al. (1997), including the most important heterogeneous reactions on sulphate and PSC aerosols. The ULAQ model also includes the most important 
tropospheric aerosols. The size distribution of sulphate (both tropospheric and stratospheric) and PSC aerosols, treated as NAT and water ice, are calculated using a fully interactive and mass conserving microphysical code for aerosol formation and growth. Denitrification and dehydration due to PSC sedimentation are calculated explicitly from the NAT and ice aerosol predicted size distribution. WMGHG and halogen scenarios are taken from WMO (1999).

Changes in surface fluxes of $\mathrm{CO}, \mathrm{NO}_{\mathrm{x}}$, non-methane hydrocarbons, $\mathrm{SO}_{\mathrm{x}}$ and carbonaceous aerosols are taken from IPCC (2001). Climatological sea surface temperatures (SSTs) are used for present day conditions, and a simple heat source model is used to estimate the change in SSTs for future conditions.

\subsection{Model ozone climatologies for the current atmosphere}

To put the subsequent model results into context, we first compare the ozone climatologies from the different models during spring with the corresponding satellite observations for the years 1993-2000 (see Rozanov et al., 2001 for a description of the data included). The geographical distribution of the monthly mean total ozone over the northern hemisphere in March and over the southern hemisphere in October are presented in Figs. 1 and 2 for the data and participating models. The hemispheric area weighted total ozone is presented on Table 2. The table also includes the pattern correlation coefficients between the observed and simulated total ozone fields, defined as the correlation coefficient between observations and model over all the grid points with equal area weighting. The GISS model results are not shown in this section, as the chemistry parameterization is a perturbation from the observed climatology.

In the northern hemisphere (Fig. 1), all the models simulate the position and intensity of the primary total ozone maximum reasonably well. However, UMETRAC, CCSR/NIES, E39/C and CMAM models slightly overestimate the extension of the ozone maximum area. In these models the area where total ozone is higher than 425 DU covers a substantial part of Siberia and spreads to northern Canada. Total ozone in MAECHAM/CHEM is higher than observed values over the entire hemisphere. The area of ozone maximum in the UIUC and ULAQ models extends to the Pacific sector, which implies an underestimated intensity of the Aleutian highs in the stratosphere. This dynamical feature also leads to the equatorward shift of the area with elevated total ozone in the Pacific sector. The CCSR/NIES model simulates very well the magnitude of the primary maximum, although its position is slightly shifted to the west. This shift, and the shape of the 400 DU contour imply that the intensity of the Aleutian maximum in the CCSR/NIES model is overestimated. The simulated position and magnitude of the elevated total ozone over northern Canada are in good agreement with observations. However, in most of the participating models this area is shifted to the north and its magnitude is slightly overesti-
Table 2. Simulated and observed area weighted total ozone and pattern correlation coefficients between total ozone fields. $\Delta$ is the quantity (model - observations)/observations

\begin{tabular}{lccc}
\hline March, NH & Total Ozone & $\Delta(\%)$ & Correlation \\
\hline Observations & 318.0 & - & - \\
UMETRAC & 339.9 & +6.9 & 0.98 \\
CMAM & 338.0 & +6.3 & 0.97 \\
MAECHAM/CHEM & 371.8 & +16.9 & 0.95 \\
E39/C & 351.2 & +10.4 & 0.98 \\
UIUC & 322.6 & +1.4 & 0.84 \\
CCSR/NIES & 326.1 & +2.5 & 0.98 \\
ULAQ & 337.6 & +6.2 & 0.85 \\
\hline October, SH & Total Ozone & $\Delta(\%)$ & Correlation \\
\hline Observations & 291.1 & - & - \\
UMETRAC & 337.8 & +16.0 & 0.86 \\
CMAM & 324.3 & +11.4 & 0.97 \\
MAECHAM/CHEM & 349.0 & +19.9 & 0.98 \\
E39/C & 301.7 & +3.6 & 0.97 \\
UIUC & 277.2 & -4.8 & 0.97 \\
CCSR/NIES & 318.2 & +9.3 & 0.95 \\
ULAQ & 314.8 & +8.1 & 0.94 \\
\hline
\end{tabular}

mated, while the UIUC and ULAQ models tend to shift this area to the south. In agreement with the observations the area with rather low total ozone appears in UIUC, CCSR/NIES, $M A E C H A M / C H E M$ and E39/C models just over the North Pole reflecting the position of the Polar vortex. However, this feature is not present in the UMETRAC, ULAQ and CMAM total ozone maps. Table 2 shows that the overall performance of all the models in March over the northern hemisphere is reasonably good. The pattern correlation with observed data is greater than 0.95 for UMETRAC, CCSR/NIES, E39/C, CMAM and MAECHAM/CHEM models but a lower correlation occurs for the UIUC and ULAQ models partially because of the underprediction in the strength of the Aleutian high. All the models overestimate the area weighted hemispheric total ozone, by on average $7.2 \%$.

Figure 2 shows the total ozone for the observations and models for the southern hemisphere in October. The pattern correlations between simulated and observed total ozone (Table 2) are similar to that in the northern hemisphere.

UMETRAC, CCSR/NIES, MAECHAM/CHEM, ULAQ and CMAM models noticeably overestimate the area weighted total ozone. This total ozone surplus comes from slightly higher total ozone in the tropics and from substantial overestimation of the magnitude of the total ozone maximum over the middle latitudes. For example the total ozone exceeds $475 \mathrm{DU}$ in the UMETRAC results, $450 \mathrm{DU}$ in MAECHAM/CHEM and 425DU in the CCSR/NIES, and CMAM results, while in the observations the total ozone peak just exceeds 375 DU. From these results we can conclude ei- 

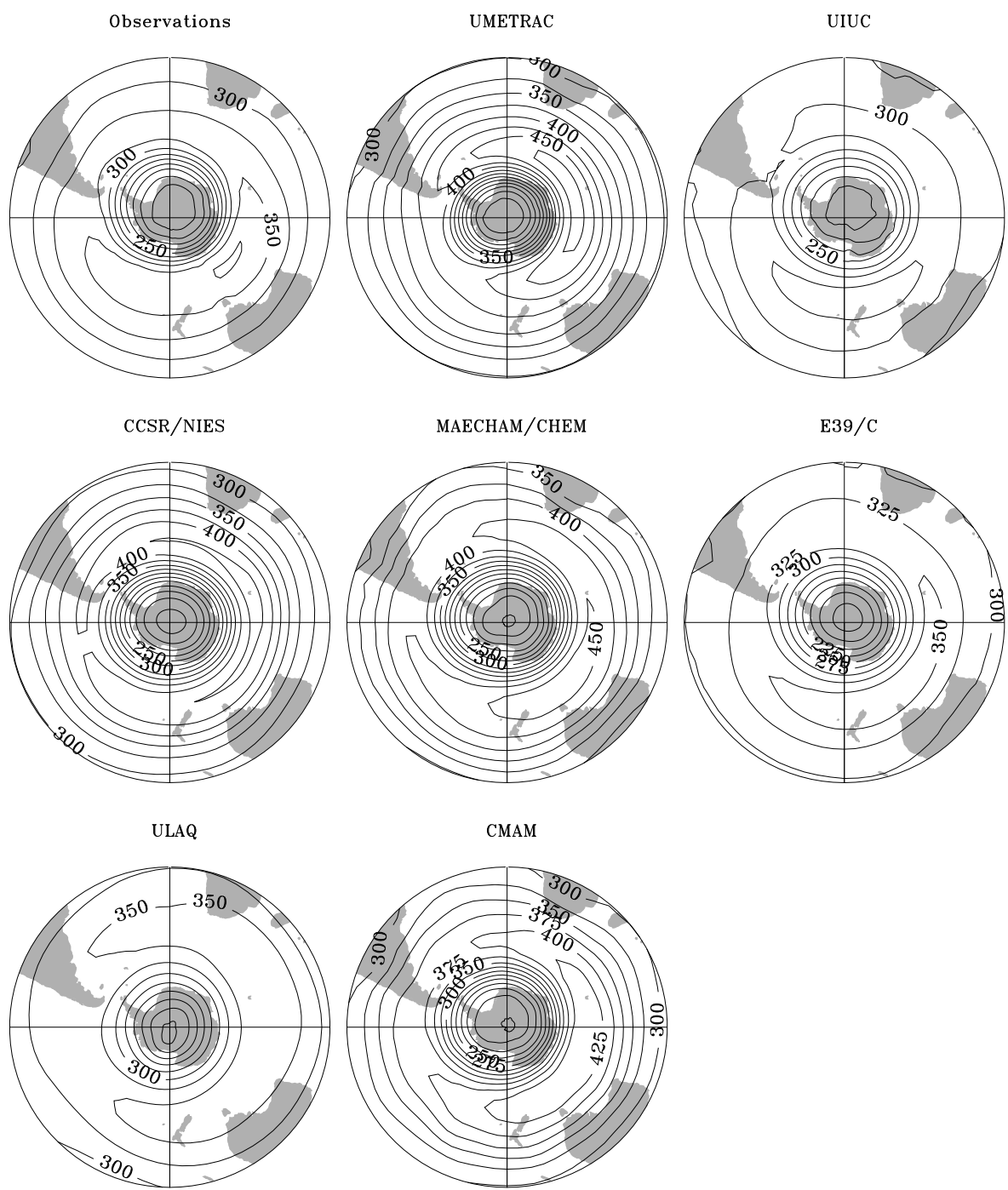

Fig. 2. As in Fig. 1, but for October in the Southern Hemisphere.

ther that the meridional transport and wave forcing are overestimated by these models, or that the vortex barrier is too strong. For MAECHEM/CHEM, CCSR/NIES and CMAM the cold-pole bias evident in Fig. 5 suggests that the latter effect is definitely a factor. In contrast the E39/C and UIUC models, which are the two models with the lowest upper boundary, match the observed total ozone very closely. The position of the total ozone maximum over middle-latitudes is also shifted from the Australian sector to the Indian Ocean sector in UMETRAC and CCSR/NIES models and to the Pacific Ocean sector in the UIUC model. The position and magnitude of the ozone hole over the South Pole is well reproduced by the models, implying that the amount of PSCs during the spring season and chemical ozone destruction are reasonably well captured by the chemical routines. This is discussed further in Sects. 3.2 and 4.4.

\section{Model uncertainties}

\subsection{Temperature biases}

Many climate models without chemistry but with a fully resolved stratosphere have a cold bias of the order of 5-10 K in high southern latitudes in the lower stratosphere, suggesting that the residual circulation is too weak (Pawson et al., 2000), i.e. there is too little downwelling in polar latitudes and too little upwelling in lower latitudes. This temperature bias could have a significant impact on model heterogeneous chemistry, and enhance ozone destruction. The "cold pole problem" extends to higher levels in the stratosphere and by the thermal wind relation gives rise typically to a polar night jet that is too strong and which has an axis that does not slope with height, whereas in the observations the polar night jet axis slopes with height towards the equator in 

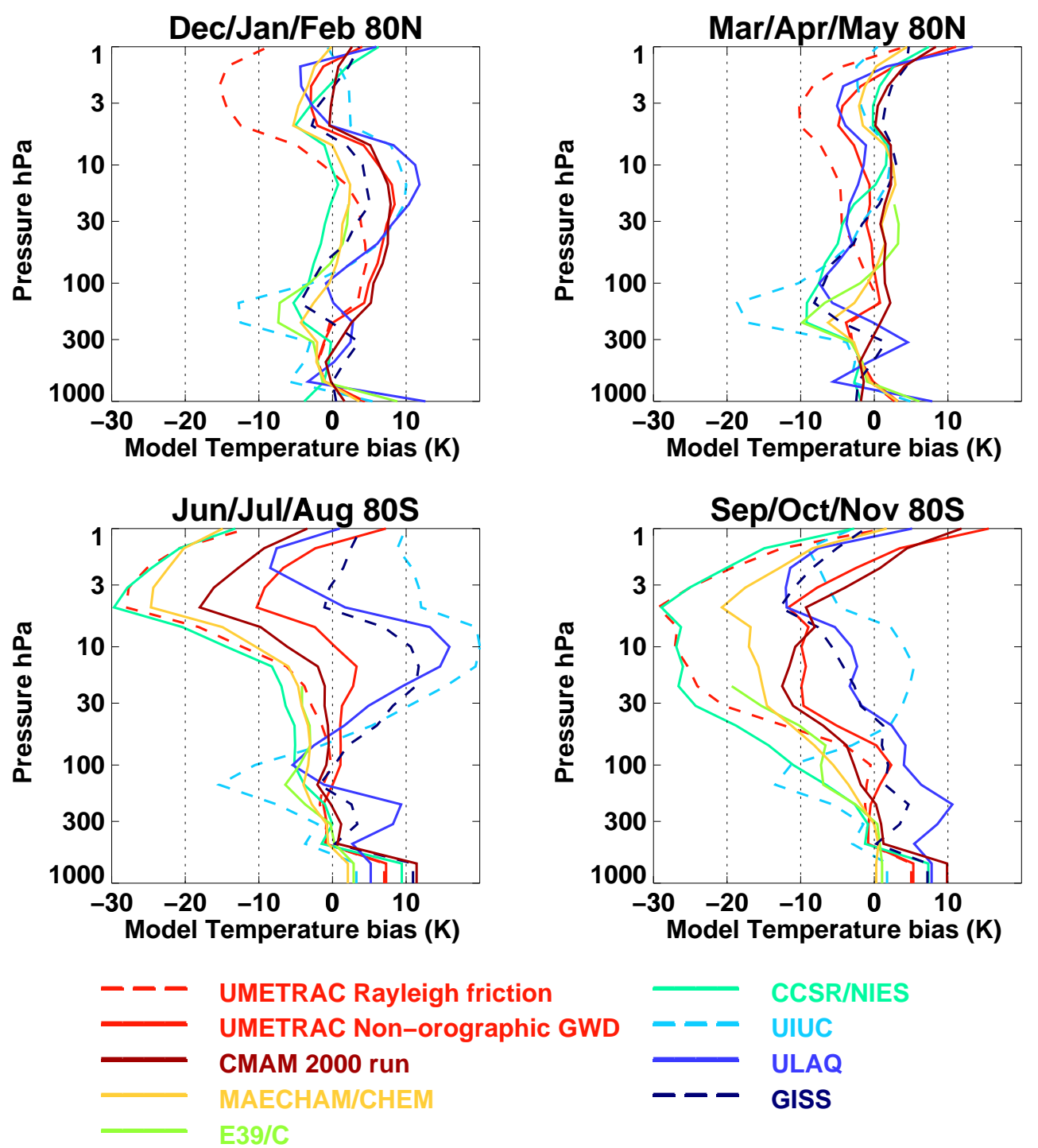

Fig. 3. Temperature biases at $80^{\circ} \mathrm{N}$ and $80^{\circ} \mathrm{S}$ for the winter and spring seasons, as a function of pressure. To determine the bias, a climatology determined from 10 years of UKMO data assimilation temperatures was subtracted from the model results averaged for the same years as indicated in Fig. 1.

the upper stratosphere. The weaker jet and slope of the axis allow waves to propagate into higher latitudes and maintain higher polar temperatures. A potentially important component of climate change is whether these waves increase in amplitude with time since this will likely affect the evolution of ozone: see Sect. 3.4. A practical solution for those models with a cold bias is to adjust the temperatures in the heterogeneous chemistry (e.g. Austin et al., 2000) so that the heterogeneous chemistry is calculated using realistic temperatures. The strong polar night jet is also associated with a vortex that breaks down later in the spring, particularly in the southern hemisphere. In a chemistry-climate model this can lead to a longer lasting ozone hole. Adjusting model PSC threshold temperatures to allow for model temperature bias cannot solve this problem.

Recently the development of non-orographic gwd schemes for climate models (Medvedev and Klaassen, 1995; Hines, 1997; Warner and McIntyre, 1999) has resulted in a significant reduction in the cold pole problem relative to simulations that rely on Rayleigh friction to decelerate the polar night jet (e.g. Manzini and McFarlane, 1998). Two of these schemes have also been shown to produce a quasi-biennial oscillation (QBO) when run in a climate model (Scaife et al., 2000, McLandress, 2002). The latest versions of several coupled chemistry-climate models now employ such schemes: CMAM uses the Medvedev-Klaassen scheme (Medvedev et al., 1998) or the Hines scheme (McLandress, 1998); the latest version of UMETRAC uses the Warner and McIntyre 

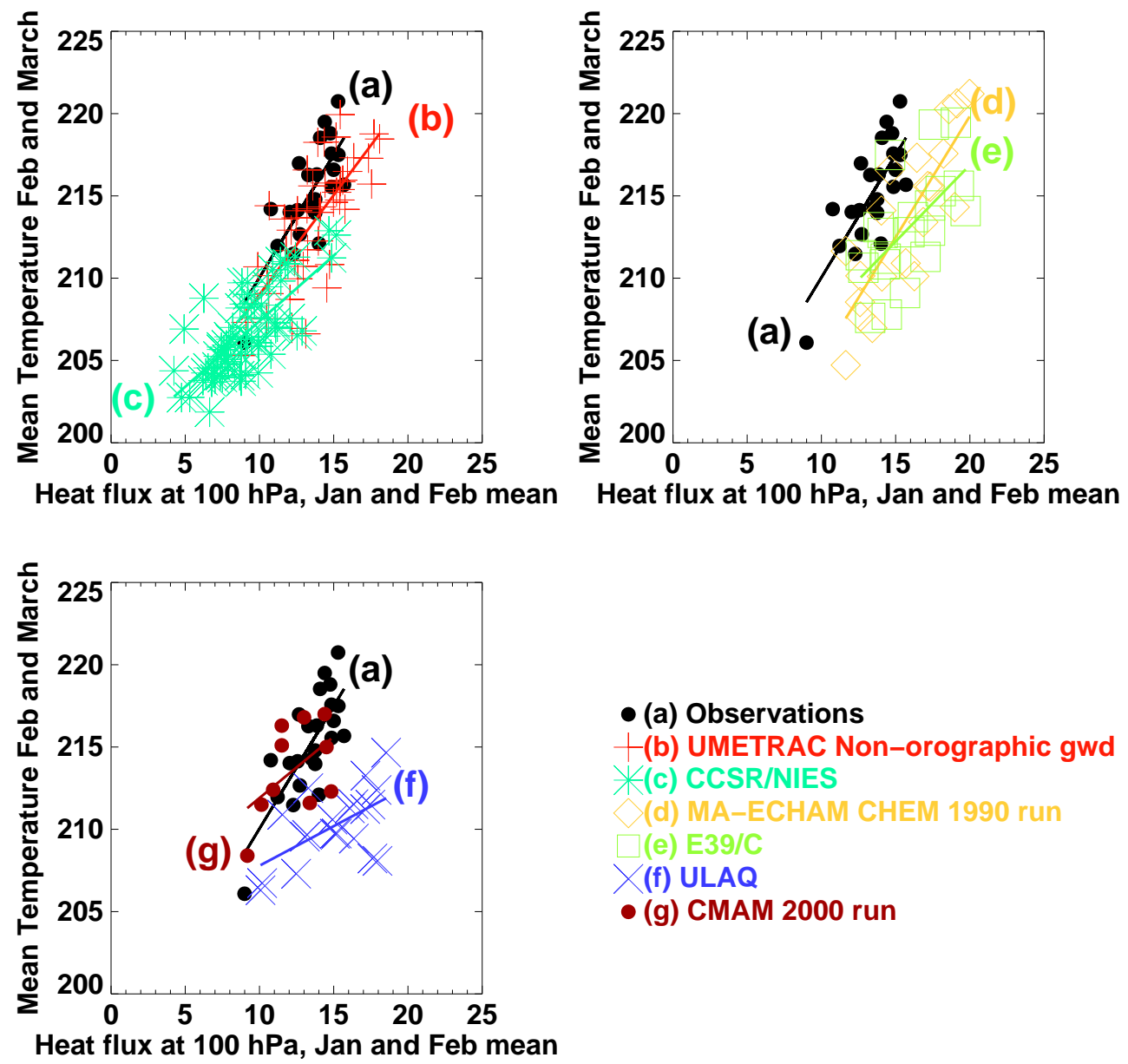

- (a) Observations

(b) UMETRAC Non-orographic gwd

(c) CCSR/NIES

(d) MA-ECHAM CHEM 1990 run

(e) $E 39 / C$

(f) ULAQ

- (g) CMAM 2000 run

Fig. 4. Scatter diagrams of heat flux $\overline{v^{\prime} T^{\prime}}$ (averaged $40^{\circ}-80^{\circ} \mathrm{N}$, at $100 \mathrm{hPa}$ for January and Febuary) against temperature (averaged $60^{\circ}-$ $90^{\circ} \mathrm{N}$, at $50 \mathrm{hPa}$ for February and March) for participating models for the same years as indicated in Fig. 1. The solid lines are linear regression lines between the two variables. The observations are taken from NCEP assimilations. The heat flux is in units of Kms ${ }^{-1}$, the temperature is in $\mathrm{K}$.

scheme; and MAECHAM/CHEM uses the Hines scheme (Manzini et al., 1997). The GISS GCM has used a nonorographic gwd scheme for many years (Rind et al., 1988a, b), which is able to reproduce high latitude temperatures reasonably well (Shindell et al., 1998b) but does not simulate a QBO in the tropics.

Figure 3 shows model temperature biases as a function of height for $80^{\circ} \mathrm{N}$ and $80^{\circ} \mathrm{S}$ for the winter and spring seasons. To determine the biases, a 10-year temperature climatology determined from data assimilation fields (Swinbank and O'Neill, 1994) was subtracted from the mean model temperature profiles applicable to the 1990s. The UKMO temperatures are considered to be typically about $2 \mathrm{~K}$ too high at low temperatures (e.g. Pullen and Jones, 1997). Although typical model biases are somewhat larger than $2 \mathrm{~K}$ it is also possible that our climatology in the high latitude regions are less reliable because of large interannual variability. The upper and middle stratospheric cold pole problem is particularly noticeable in the south in the UMETRAC (with Rayleigh fric- tion), CCSR/NIES, E39/C and MAECHAM/CHEM results. With a non-orographic gwd scheme the temperature biases reduce considerably and both CMAM and UMETRAC have very similar results in the seasons analysed. In the UIUC, GISS and ULAQ models a warm bias is present in the middle stratosphere in winter. For the latter models, which have lower horizontal resolution than the other five, the biases may be a result of the need to adjust physical parameterizations to obtain improved climatologies and that such adjustments are not always suitable for all seasons. The ULAQ model, for example, includes vertical diffusion, in addition to Rayleigh friction. The MAECHAM/CHEM model uses the Hines nonorographic gwd scheme, but in southern winter the results are only a slight improvement on the Rayleigh friction results of UMETRAC and CCSR/NIES. For other seasons, the MAECHAM/CHEM temperature bias is much smaller and is similar to the results of E39/C which has equivalent physics and chemistry, but does not have a non-orographic gwd scheme. Nonetheless, Manzini et al. (1997) indicated 


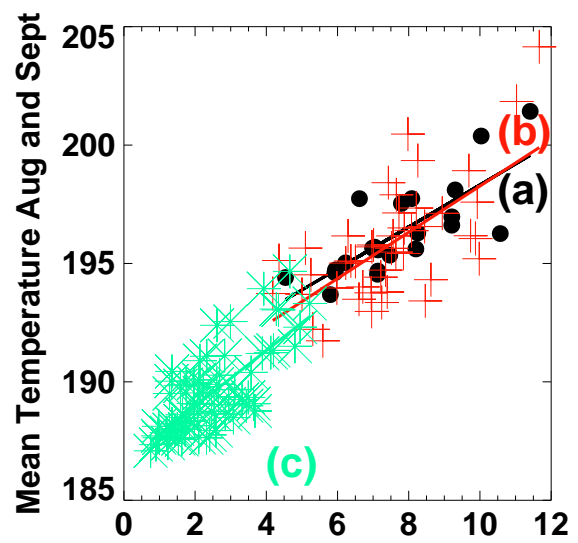

Heat flux at $100 \mathrm{hPa}$, Jul and Aug mean

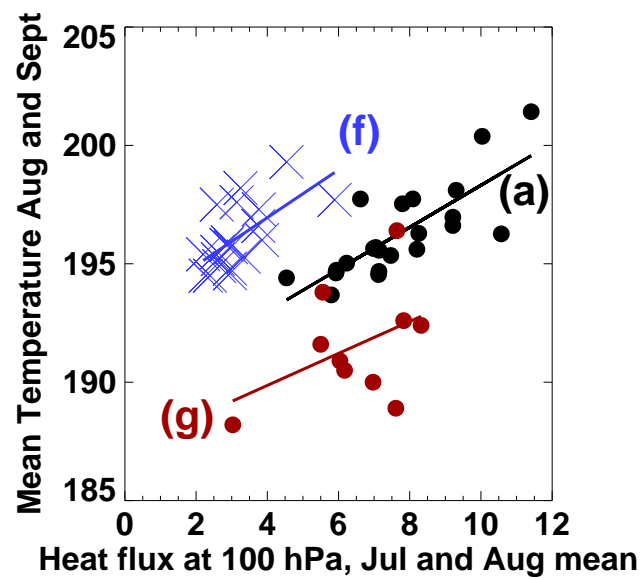

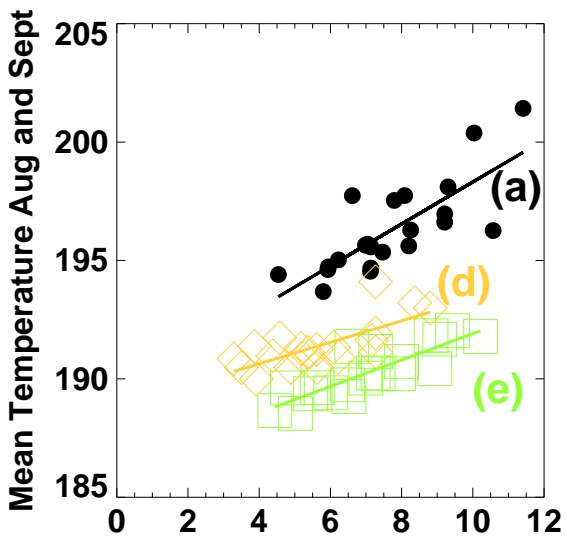

Heat flux at $100 \mathrm{hPa}$, Jul and Aug mean
- (a) Observations

+ (b) UMETRAC Non-orographic gwd

(c) CCSR/NIES

(d) MA-ECHAM CHEM 1990 run

(e) E39/C

(f) ULAQ

(g) CMAM 2000 run

Fig. 5. As in Fig. 4, but for the Southern Hemisphere for the months July and August, and August and September, respectively. For convenience, the usual convention for $v$ has been used (i.e. negative values indicate toward the South Pole) but the values have been multiplied by -1$)$.

that the temperature biases in the core climate model are smaller when non-orographic gwd is included.

At $80^{\circ} \mathrm{N}$ temperature biases are generally somewhat smaller than at $80^{\circ} \mathrm{S}$ and for some models are positive at some levels. The northern lower stratospheric temperature biases would generally lead to insufficient heterogeneous ozone depletion in early winter but excessive ozone depletion in the more important spring period. In the southern hemisphere, spring cold biases could lead to more extensive PSCs than observed and delayed recovery in Antarctic ozone.

Newman et al. (2001) showed that the lower stratospheric temperature is highly correlated with the lower stratospheric heat flux $\overline{v^{\prime} T^{\prime}}$ slightly earlier in the year. The heat flux provides a measure of the wave forcing from the troposphere, and in Fig. 4, the heat flux at $100 \mathrm{hPa}$ averaged over the domain $40^{\circ}-80^{\circ} \mathrm{N}$ for January and February is plotted against temperature averaged over the domain $60^{\circ}-90^{\circ} \mathrm{N}$ at $50 \mathrm{hPa}$ for February and March. Similar results for the southern hemisphere are shown in Fig. 5. Newman et al. choose the period of the temperature average as 1-15 March, which maximises the correlation coefficient between the two variables at 0.85 . However, here we choose a longer period for the temperature average to smooth over any model and atmospheric transients. This reduces the correlation coefficient for NCEP data only slightly, to 0.77 (0.78 in the south). Table 3 shows the correlation coefficient $(R)$ between the two variables, computed for data and model results within the period 1980-2000. $T_{0}$ is the temperature intercept at zero flux, $\beta$ is the gradient of the lines in Figs. 4 and 5, and $\sigma$ is the standard deviation in the computation of $\beta . T_{0}$ indicates the radiative equilibrium temperature, including a contribution from small scale waves not represented in the heat flux (e.g. Newman et al., 2001).

In the north (Fig. 4), the model results are for the most part in reasonable agreement with the observations, although the model correlation coefficients vary in the range 0.52 to 0.86. Model horizontal resolution may have significantly affected the results: in general the model regression lines are less steep (smaller $\beta$ in Table 3 ) as the model resolution decreases. This could be because while low-resolution models 
Table 3. Statistical analysis of the linear regression between the area averaged temperature $(\mathrm{K})$ at $50 \mathrm{hPa}$ polewards of $60^{\circ} \mathrm{N}$ for February and March, and the heat flux $\left(\mathrm{Kms}^{-1}\right)$ at $100 \mathrm{hPa}$ between $40^{\circ}$ and $80^{\circ} \mathrm{N}$ for January and February (Northern Hemisphere). The Southern Hemisphere results are for the months August and September and July and August, respectively. $R$ is the correlation coefficient between the variables, $T_{0}$ is the intercept of the line at zero heat flux, $\beta$ is the gradient of the line and $\sigma$ is the standard error in the computation of $\beta$

\begin{tabular}{l|rrrr|rrrrr}
\hline \multicolumn{1}{c}{ Model/Observations } & \multicolumn{1}{c}{ N'ern Hemi. } & \multicolumn{1}{c}{ S'ern Hemi. } \\
& $R$ & $T_{0}$ & $\beta$ & $\sigma$ & $R$ & $T_{0}$ & $\beta$ & $\sigma$ \\
NCEP (Observations) & 0.77 & 195.1 & 1.49 & 0.27 & 0.78 & 189.4 & 0.89 & 0.16 \\
UMETRAC Non-orographic gwd & 0.77 & 196.9 & 1.20 & 0.22 & 0.74 & 187.5 & 1.14 & 0.23 \\
$\quad$ Rayleigh Friction & 0.63 & 196.7 & 1.19 & 0.33 & 0.47 & 186.8 & 0.82 & 0.34 \\
CMAM 2000 & 0.52 & 204.5 & 0.75 & 0.43 & 0.44 & 191.3 & 0.68 & 0.50 \\
MAECHAM/CHEM 1990 & 0.83 & 190.4 & 1.47 & 0.23 & 0.72 & 188.8 & 0.45 & 0.10 \\
E39/C & 0.62 & 198.3 & 0.93 & 0.28 & 0.86 & 186.3 & 0.56 & 0.08 \\
CCSR/NIES & 0.86 & 199.2 & 0.86 & 0.14 & 0.66 & 187.4 & 0.98 & 0.29 \\
ULAQ & 0.58 & 203.0 & 0.48 & 0.16 & 0.64 & 192.9 & 1.01 & 0.29 \\
\hline
\end{tabular}

can capture the low-amplitude wave, small heat flux case, they may have more difficulty capturing the large heat flux case with its possibly significant potential enstrophy cascade to larger wavenumbers. However, this covers a broad spectrum of results. For example, CMAM simulates a wide range of heat fluxes consistent with observations but the functional relationship with temperature is highly sensitive to a single year's data. Removal of the single point corresponding to the highest heat flux, increases the correlation coefficient to 0.67 and increases $\beta$ to 1.06 , much closer to that expected on the basis of the model resolution. This implies that the 10year CMAM run is too short to give fully meaningful results in the northern hemisphere for this diagnostic. The performance of the models might also depend on the dissipation that the models have at short spatial scales, although this is more difficult to compare.

In the southern hemisphere (Fig. 5) the correlation coefficients cover the range 0.44 to 0.86 , wider than in the north. $T_{0}$ is considerably lower, probably due to lower ozone amounts, and all the models agree well with the observed $T_{0}$. For the time slice experiments (e.g. MAECHAM/CHEM) $T_{0}$ decreased from the 1960 results (not shown) to the 1990 results due to the WMGHG increase and ozone depletion. The values of $\beta$ are also generally smaller in the southern hemisphere, except for the CCSR/NIES and ULAQ models. Unlike in the northern hemisphere, the model results indicate that resolution is not a factor in the value of $\beta$. That is, both low and high resolution models are in principle capable of capturing the observed temperature-heat flux relationship.

\subsection{The simulation of polar stratospheric clouds}

During the last few years, considerable progress has been made regarding the understanding of PSCs and the associated heterogeneous chemical reactions. Some of these developments are discussed in Carslaw et al. (2001) and WMO (2002), Chapter 3. They include the observation of many different types of PSCs, both liquid and solid forms, and the laboratory measurement of a wide range of chemical reaction rates as summarised in Sander et al. (2000). Coupled chemistry-climate models have a variety of PSC schemes with and without sedimentation, but as indicated in Sect. 3.1, some models have large climatological biases in the polar regions. If the models are to produce accurate simulations of ozone depletion, the temperature field must give realistic distributions near the PSC temperature threshold. So that common ground can be established between models, we here use the temperature at $50 \mathrm{hPa}$ as an indicator, hence ignoring the impact of $\mathrm{HNO}_{3}$ and sulphate concentrations on the determination of PSC surface areas.

Following Pawson and Naujokat (1997) and Pawson et al. (1999), Fig. 6 shows for each of the participating models and observations the time integral throughout the winter of the approximate PSC area at $50 \mathrm{hPa}$, as given by the areas within the $195 \mathrm{~K}$ and $188 \mathrm{~K}$ temperature contours. This is the quantity $\tilde{A}_{\tau}$ indicated in Fig. 9 of Pawson et al. (1999), but without the normalisation factor of $1 / 150$, for $\tau_{N A T}=195 \mathrm{~K}$ and $\tau_{I C E}=188 \mathrm{~K} . \tilde{A}_{\tau}$ is here measured in terms of the fraction of the hemisphere covered in $\%$ times their duration in days. For the ice amount in the Arctic, $\tilde{A}_{\tau}$ varies dramatically between zero (ULAQ and CMAM models, not shown) and 215 (CCSR/NIES) in units of \% of the hemisphere times days and the models have large interannual variability. Arctic NAT also covers a large range, both for different models and in the interannual variability for each simulation. In accordance with their temperature biases, several models have larger areas of NAT than are typically derived from observations. The ULAQ PSCs are in good agreement with observations, despite a slight temperature bias, while UMETRAC and CMAM have lower PSCs than are derived from observations. In the Antarctic most models have much lower fractional interannual variation, but again the results for separate models cover an exceedingly large range for the ice amount. ULAQ and UMETRAC are in good agreement with obser- 

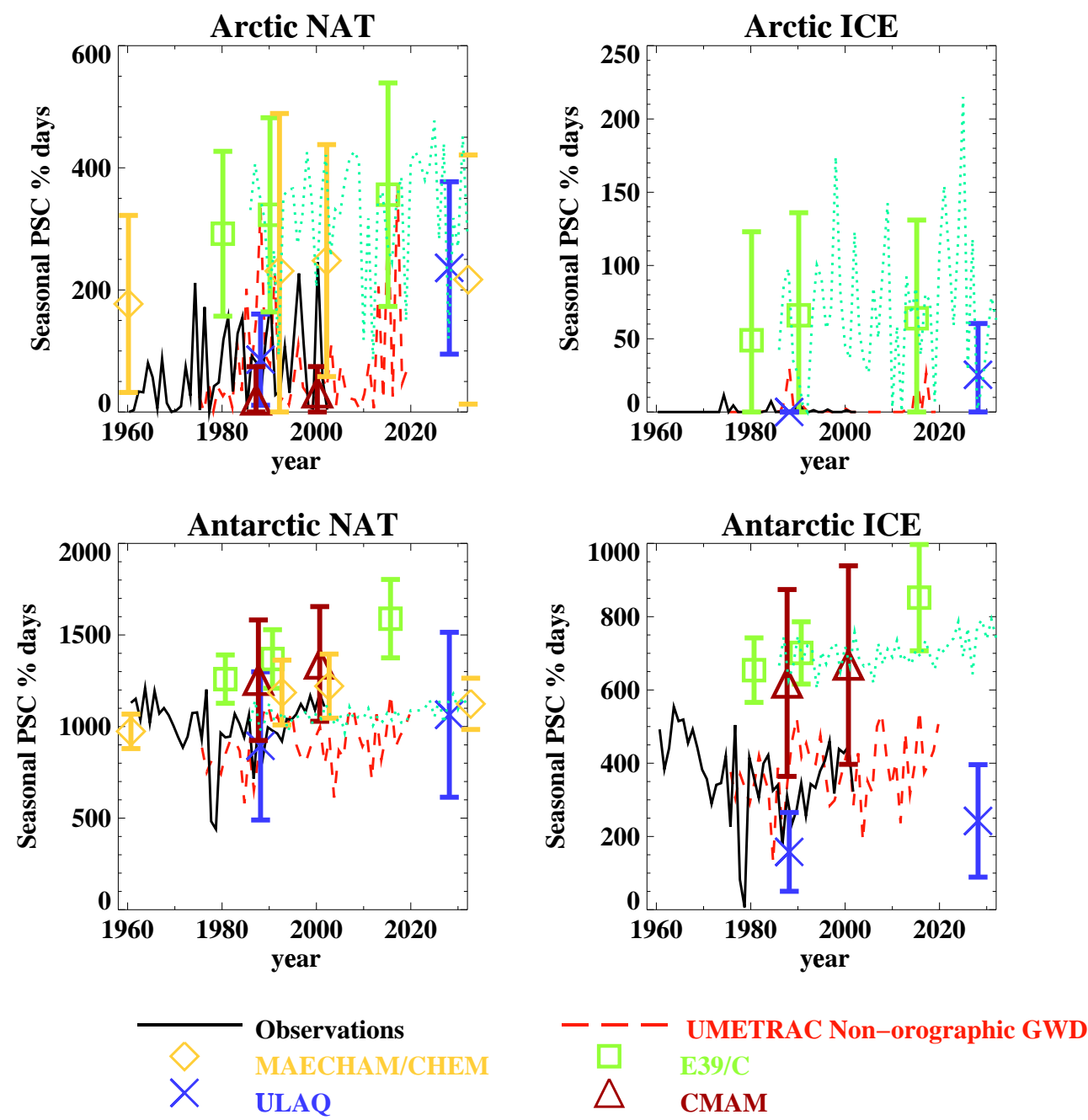

Fig. 6. Seasonal integration of the hemispheric area at $50 \mathrm{hPa}$, corresponding to the temperatures below $195 \mathrm{~K}$ (approximate NAT temperature) and below $188 \mathrm{~K}$ (approximate ICE temperature) for participating models except E39/C which shows the actual PSC area. The error bars for the time slice runs indicate $95 \%$ confidence intervals. For clarity, ULAQ data are plotted two years early, and for 1990 and 2030 MAECHEM CHEM data are plotted two years late.

vations for both the NAT and ice temperature, but the other models tend to overpredict the PSC amounts. The implications for ozone on the differences between the models is discussed further in Sect. 4.4.

The sedimentation of PSC particles is now thought to be important in polar ozone depletion (e.g. Waibel et al., 1999), but is absent from many coupled chemistry-climate models. Using a 3-D model without transport, Waibel et al. (1999) suggested that cooling associated with greenhouse gas increases may lead to higher ozone depletion than if sedimentation were ignored. There are many practical difficulties associated with incorporating sedimentation in climate models. The full details, including dividing the particles into different sized bins (e.g. Timmreck and Graf, 2000), cannot be readily simulated in most models as this would require the transport of particles covering a large number of size ranges, which may exceed the total number of tracers transported. While the ULAQ model (Pitari et al., 2002) is able to simulate this level of complexity, the model resolution is somewhat coarse (see Table 1). Most models therefore adopt some extremely simplified procedures. For example in the UMETRAC model (Austin, 2002) sedimentation is assumed to occur for NAT or ice particles of fixed size. The vertical transport equation is solved locally to give a sedimented field which is not explicitly advected horizontally but is tied to the field of a conserved horizontal tracer. Other models (e.g. Egorova et al., 2001; Hein et al., 2001; Pitari et al., 2002) transport the sedimented field in more detail, but the accuracy of this transport may be limited by model spatial resolution, and whether it can treat small scale features with high enough precision. 
DJF
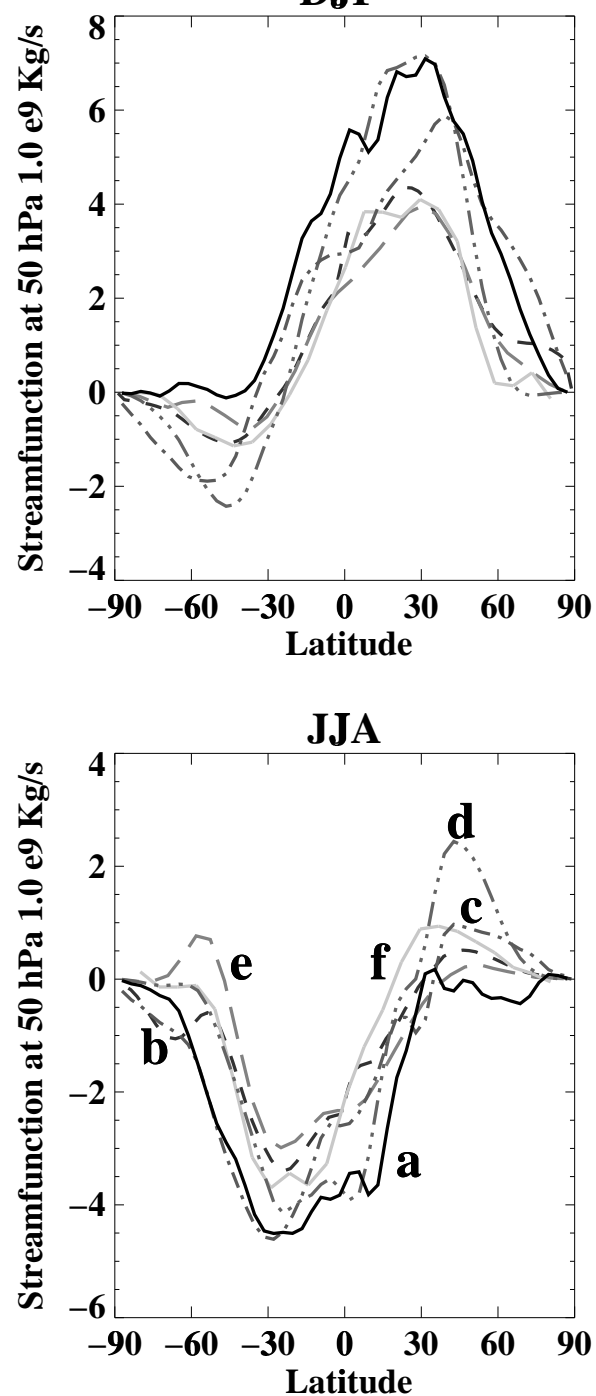

(a) Observations

(c) MAECHAM/CHEM

(e) CCSR/NIES
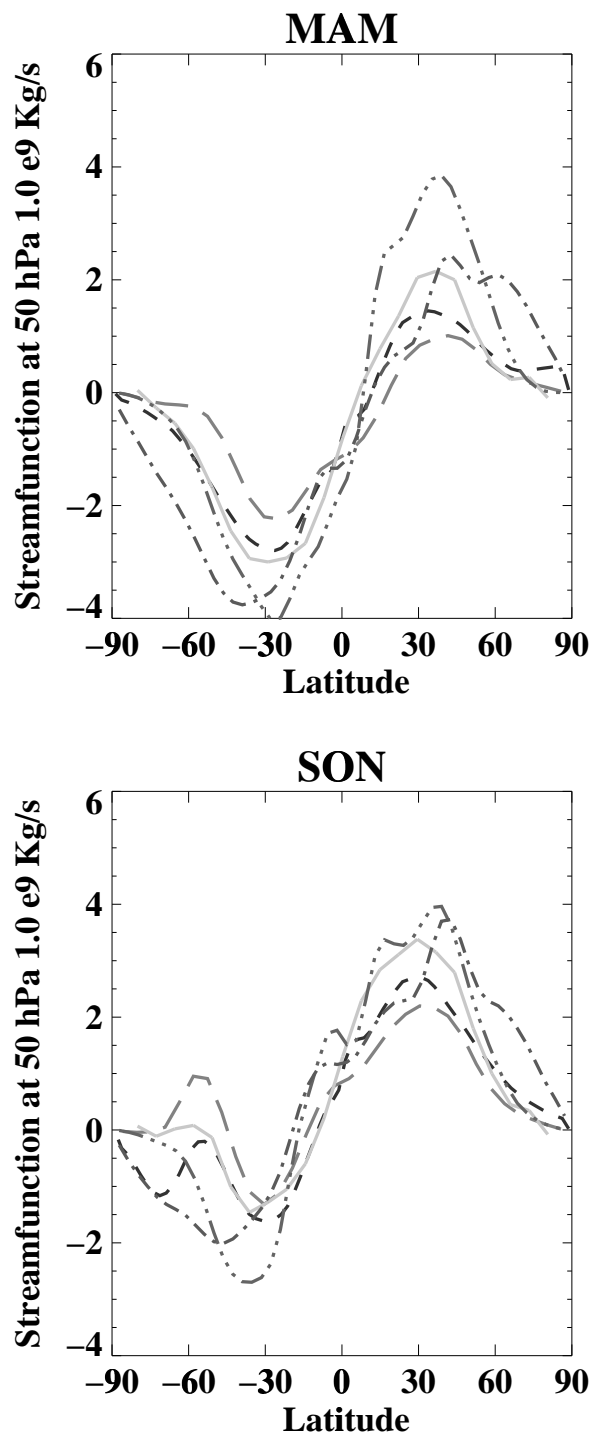

(b) UMETRAC

(d) $\mathrm{E} 39 / \mathrm{C}$

Fig. 7. Residual streamfunction $\left(10^{9} \mathrm{Kgs}^{-1}\right)$ at $50 \mathrm{hPa}$ as a function of latitude and season for the participating models.

Recently, the presence of large, nitric acid containing particles exceeding $10 \mu \mathrm{m}$ in diameter has been detected in the lower stratosphere (Fahey et al., 2001). Models have only just started to take the production of these particles into account (Carslaw, personal communication, 2002), although they may have an important impact on ozone depletion.

\subsection{The transport of constituents}

There is strong evidence from a number of modelling studies (Garcia and Boville, 1994; Shepherd et al., 1996; Lawrence, 1997; Austin et al., 1997; Rind et al., 1998; Beagley et al., 2000) that the position of the model upper boundary can play a significant role in influencing transport and stratospheric dynamics due to the "downward control principle" (Haynes et al., 1991). The sensitivity of the dynamical fields to the position of the upper boundary may be more when using non-orographic gwd schemes than when Rayleigh friction is used, although if all the non-orographic gwd that is produced above the model boundary is placed instead in the top model layer, this sensitivity reduces (Lawrence, 1997). Model simulations with an upper boundary as low as $10 \mathrm{hPa}$ have been completed (e.g. Hein et al., 2001; Schnadt et al., 2002; Dameris et al., 1998). Schnadt et al. (2002) show the meridional circulation of the DLR model and this gives 
the expected upward motion from the summer hemisphere and downward motion over the winter hemisphere, although modelled meridional circulations are known to extend into the mesosphere (e.g. Butchart and Austin, 1998). From the results of Schnadt et al. (2002) it could be implied that it is less important for total ozone to have a high upper boundary, but more important to have high resolution in the vicinity of the tropopause. At present the evidence appears ambiguous: for example in the total ozone presented by Hein et al. (2001) insufficient ozone is transported to the North Pole, but there is excessive subtropical ozone transport. This could be related to the cold pole problem, rather than the position of the upper boundary. While the transport effect on ozone is direct, other considerations are the transport of long-lived tracers such as $\mathrm{NO}_{\mathrm{y}}$ and water vapour which have a photochemical impact on ozone. Consequently, it is generally recognised that the upper boundary should be placed at least as high as $1 \mathrm{hPa}$ (e.g. Rozanov et al., 2001; Pitari et al., 2002) with many models now placing their boundary at about $0.01 \mathrm{hPa}$ (e.g. Shindell et al., 1998a; Austin et al., 2001; Steil et al., 2002; Nagashima et al., 2002). In comparison, CMAM (de Grandpré et al., 2000) has an upper boundary somewhat higher (c. $0.0006 \mathrm{hPa}$ ) to allow a more complete representation of gwd to reduce the cold pole problem (Sect. 3.1) and to simulate upper atmosphere phenomena.

The differences in transport can be assessed by investigating the Brewer-Dobson circulation computed for the individual models. An indicator of the circulation is given by the residual mean meridional circulation

$\bar{v}^{*}=\bar{v}-\frac{\partial}{\partial p}\left(\frac{\overline{v^{\prime} \theta^{\prime}}}{\partial \bar{\theta} / \partial p}\right)$,

$\bar{\omega}^{*}=\bar{\omega}+\frac{1}{a \cos \phi} \frac{\partial}{\partial \phi}\left(\cos \phi \frac{\overline{v^{\prime} \theta^{\prime}}}{\partial \bar{\theta} / \partial p}\right)$

For details of the notation see Edmon et al. (1980). The residual circulation $\left(\bar{v}^{*}, \bar{\omega}^{*}\right)$ is then used to compute the residual streamfunction $\Psi$, defined by

$$
\frac{\partial \Psi}{\partial \phi}=-a \cos \phi \bar{\omega}^{*}, \frac{\partial \Psi}{\partial p}=\cos \phi \bar{v}^{*}
$$

Figure 7 shows the mass streamfunction $F_{m}=2 \pi a \Psi / g$ at $50 \mathrm{hPa}$ as a function of latitude for the participating models, computed by integrating the second equation downwards with $\Psi=0$ at $p=0$. The observations in the figure are based on ERA-15 for the period 1979 to 1993 (Gibson et al., 1997). The spatial resolution of the original fields was T106 with 31 vertical hybrid levels, equivalent to a grid resolution of $1.125^{\circ}$ or about $125 \mathrm{~km}$. The temporal resolution is $6 \mathrm{~h}$. Note that the ERA data were computed using a GCM with an upper boundary at $10 \mathrm{hPa}$ which may be a limiting factor in representing real variability at $30 \mathrm{hPa}$ and above. The ERA mass streamfunction is a climatological mean over the 15 analysis years. For the solstice periods, all the models have the same qualitative shape as the observations with a peak in absolute values occurring in the subtropics of the winter hemisphere. In the northern winter, the results of E39/C agree well with observations between $0^{\circ}$ and $45^{\circ} \mathrm{N}$ but farther north the streamfunction reduces significantly, implying less downward transport. The MAECHAM/CHEM results agree better with observations in high northern latitudes, but there is no indication that this is a result of the higher boundary since the other models are more consistent with the values of the E39/C model in this regard. In the extra-tropics of the northern summer, the E39/C results are higher than observations and the other models, implying too much transport.

Most models have the same problem, but to a varying extent in the southern summer. As in the northern winter, the streamfunctions of most models are smaller then observed in the southern winter, again indicating insufficient transport. Thus, the differences in the streamfunction between the MAECHAM/CHEM and E39/C results are not significantly more than between the other models. These two models have equivalent physics and chemistry, but the E39/C model has a lower upper boundary, with appropriate upper boundary condition for constituents and additional dissipation in the top layers. Thus, the dissipation may be more important than the position of the upper boundary in the determination of the residual circulation. During the equinox periods, the models are broadly consistent with each other. The E39/C model has slightly higher values of the streamfunction in mid-latitudes than other models, but this may be related to an extended winter regime in the model rather than direct effects of the upper boundary.

\subsection{Future predictions of planetary waves and heat fluxes}

In some GCMs, there is a significant trend in planetary wave propagation with time. In the GISS GCM, planetary waves are refracted equatorward as greenhouse gases increase (Shindell et al., 2001) while in the ULAQ model there is a marked reduction in the propagation of planetary waves 1 and 2 to high northern latitudes in the doubled $\mathrm{CO}_{2}$ climate simulated by Pitari et al. (2002). The latest results of the ULAQ model are different to the earlier results based on a much simpler model. It is probable that the model results are sensitive to the changes in static stability in the middle and lower troposphere with an increase in stability from increases in WMGHGs giving rise to an Arctic vortex that is less vulnerable to stratospheric warmings. In the GISS model the impact of changed planetary wave drag is largest during winter when the polar night jet is enhanced (Shindell et al., 1998a; Rind et al., 1998). Planetary wave refraction is governed by wind shear, among other factors, so that enhanced wave refraction occurs as the waves coming up from the surface approach the area of increased wind. They are refracted by the increased vertical shear below the altitude of the maximum wind increase. 

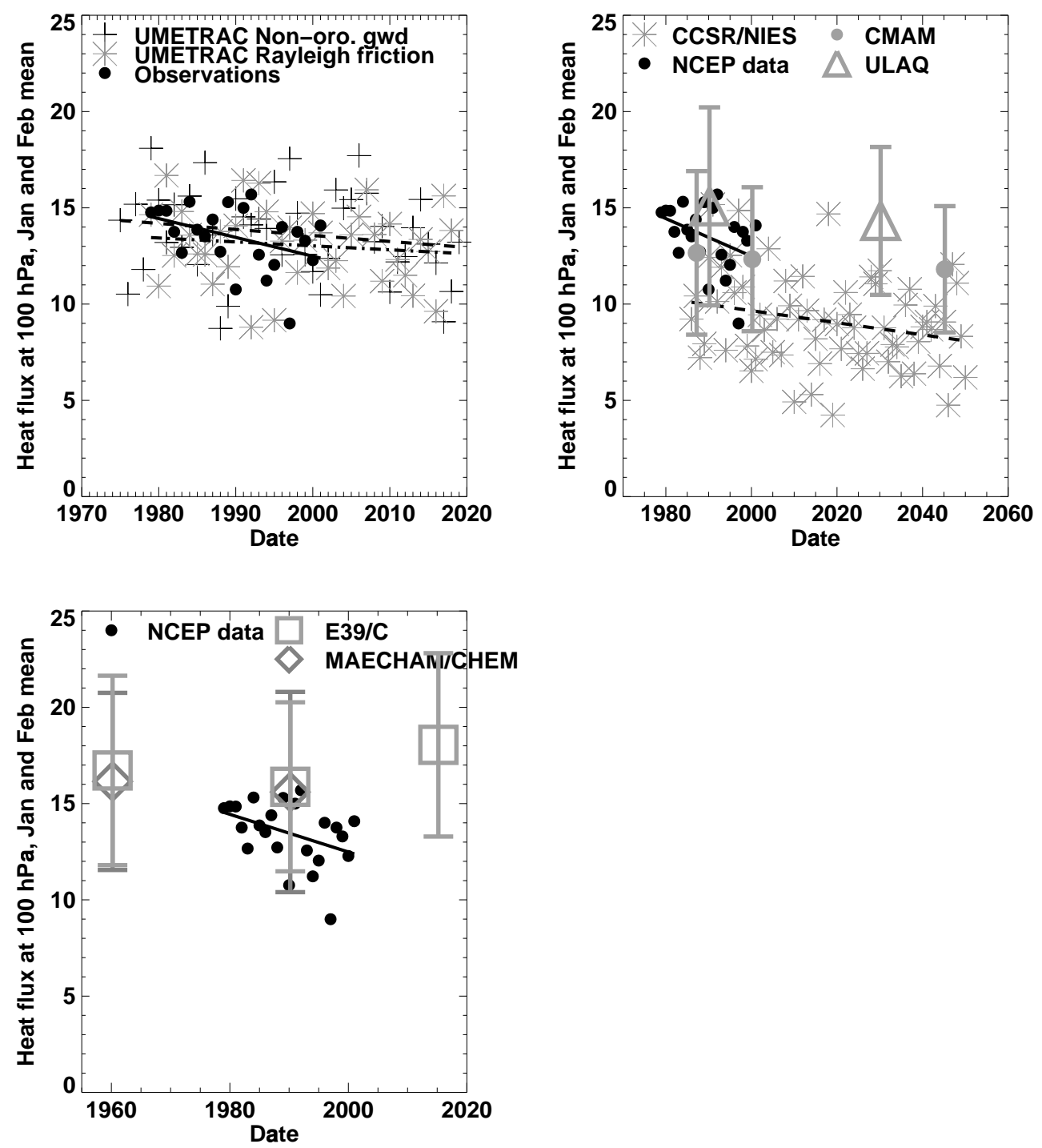

Fig. 8. Scatter diagrams of heat flux $\overline{v^{\prime} T^{\prime}}$ (averaged $40^{\circ}-80^{\circ} \mathrm{N}$, at $100 \mathrm{hPa}$ for January and February) against year for participating models. In all panels, the linear regression line between the NCEP derived heat flux and time is drawn as a solid line. Upper left panel: the dashed line is the linear regression for the non-orographic gwd run of UMETRAC, and the dot-dash line is the linear regression for the Rayleigh friction run of UMETRAC. Upper right panel: the dashed line is the linear regression for the CCSR/NIES results. Two standard deviations of the annual values are indicated by the error bars for the time slice experiments. For CMAM, the results are plotted for 2045 rather than 2028 since the results are dependent largely on the WMGHG concentrations (see Sect. 2.1).

Equatorward refraction of planetary waves at the lower edge of the wind anomaly leads to wave divergence and hence an acceleration of the zonal wind in that region. Over time, the wind anomaly itself thus propagates downward within the stratosphere (cf. Baldwin and Dunkerton, 2001) and subsequently, from the tropopause to the surface in this model.

At high latitudes in the lower stratosphere the direct radiative cooling by greenhouse gases and less radiative heating by ozone due to chemical depletion causes an increase in the strength of the polar vortex. Planetary wave changes may be a feedback which strengthens this effect. One proposed plan- etary wave feedback mechanism (Shindell et al., 2001) works as follows: tropical and subtropical SSTs increase, leading to a warmer tropical and subtropical upper troposphere via moist convective processes. This results in an increased latitudinal temperature gradient at around $100-200 \mathrm{hPa}$, leading to enhanced lower stratospheric westerly winds, which refract upward propagating tropospheric planetary waves equatorward. This results in a strengthened polar vortex.

Other model simulations show qualitatively similar effects on planetary wave propagation (Kodera et al., 1996; Perlwitz et al., 2000). Also, the E39/C results (Schnadt et al., 2002) show a small decrease in planetary wave activity for 
the period 1960 to 1990 . However, results for future simulations differ depending on the model. Without chemical feedback, the UM (Gillett et al., 2002) predicts a future increase in overall generation of planetary waves.

This leads to a greater wave flux to the Arctic stratosphere, and is even able to overcome the radiatively induced increase in the westerly zonal wind so that the overall trend is to weaker westerly flow. This also occurs in E39/C with chemical feedback (Schnadt et al., 2002). A measure of the wave activity is the high latitude heat flux which in the UM with chemical feedback (UMETRAC) is downwards throughout the period 1975-2020 (Fig. 8), giving a trend (not statistically significant) of $-2.2 \pm 3.7 \% /$ decade $(2 \sigma)$. The CCSR/NIES model, of lower resolution than UMETRAC, has systematically lower heat fluxes and also shows a downward trend during the period 1986-2050 of $-3.0 \pm$ $3.0 \% / d e c a d e$, which is marginally statistically significant. For the shorter period 1986-2001, the trend is much larger $(-6.7 \pm 13.5 \% /$ decade $)$, but the short model record reduces the statistical significance. In the time slice experiments CMAM, MAECHAM/CHEM and ULAQ the high latitude heat flux decreases throughout the periods 1987$2045,1960-1990$ and $1990-2030$ at $1.1 \pm 2.3 \% /$ decade, $1.2 \pm 3.4 \% /$ decade and $1.2 \pm 4.2 \% /$ decade $(2 \sigma)$, respectively, but none of these results are statistically significant. The decrease in the heat flux in the E39/C results is $1.7 \pm$ $1.5 \% /$ decade for $1960-1990$, which is marginally statistically significant. Thus, despite the large errors in the determination of the trends, most models agree in showing a gradual long-term weakening of the past lower stratospheric heat fluxes. For the future, most models continue to show a slight decrease, but the E39/C model shows a significant upward trend of $5.5 \pm 1.9 \% /$ decade. Thus E39/C planetary wave activity follows that of the ozone change with decreases in the past and an increase into the future.

A number of observational studies have shown that during the last twenty years the Arctic vortex has strengthened (Tanaka et al., 1996; Zurek et al., 1996; Waugh et al., 1999; Hood et al., 1999). Likewise, a trend toward equatorward wave fluxes has also occurred (Kodera and Koide, 1997; Kuroda and Kodera, 1999; Ohhashi and Yamazaki, 1999; Baldwin and Dunkerton, 1999; Hartmann et al., 2000) and the total amount of wave activity entering the stratosphere has decreased over the past 20 years (Newman and Nash, 2000; Randel et al., 2002).

The observed trend in the heat flux for the period 19792001, shown in Fig. 8 is $-6.8 \pm 6.7 \% /$ decade, just statistically significant and somewhat larger than all the model results presented here, except for the CCSR/NIES results which have a very large uncertainty. The CCSR/NIES results are somewhat smaller for the future, as indicated above, and in addition to the large errors these results suggest that extrapolation of the current observed trends to the future must remain uncertain. (a) Transient runs

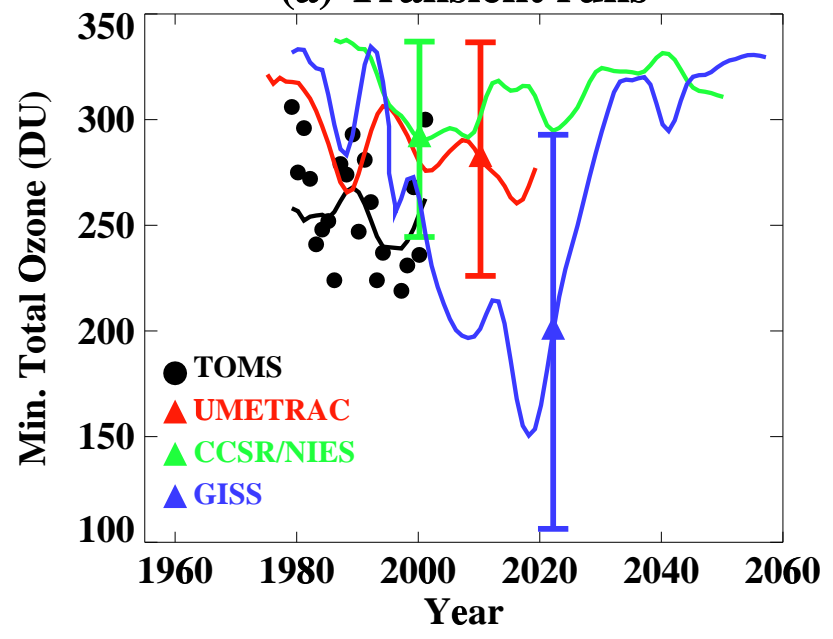

(b) Timeslice runs

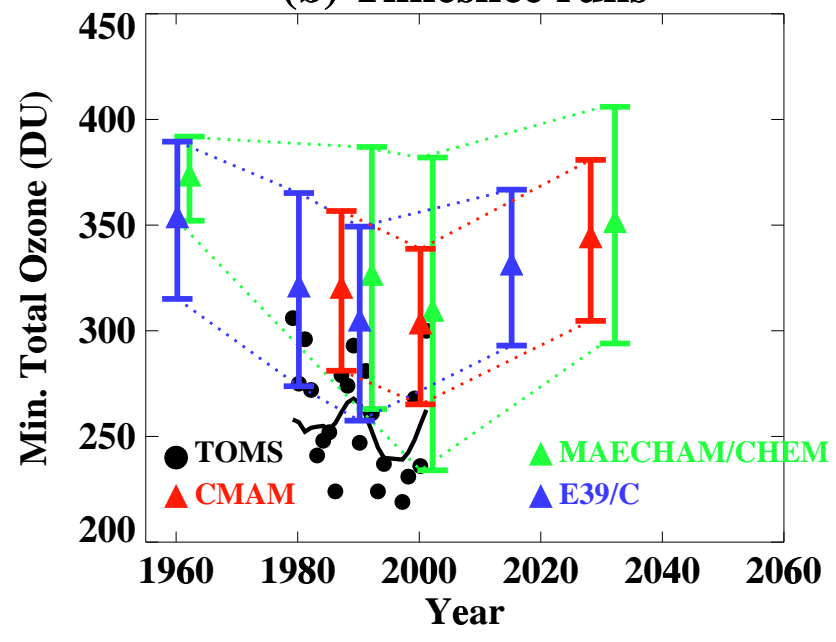

Fig. 9. Minimum Arctic (March/April) total ozone for the main experiments of this assessment. (a) Transient runs in comparison with TOMS data. The solid lines show the results of a guassian smoother applied to the individual year's results. The error bars denote twice the standard deviation of the individual years from the smoothed curve. (b) Time slice runs in comparison with TOMS data. The error bars denote the mean and twice the standard deviation of the individual years within each model sample (10 years for CMAM, 20 years for MAECHAM/CHEM and E39/C). Dotted lines are drawn between the end points of the error bars to assist in estimating trends by eye. For MAECHAM/CHEM only: (i) the values have been plotted two years late for clarity, (ii) a standard tropospheric column of $100 \mathrm{DU}$ has been added to the computed columns above $90 \mathrm{hPa}$. Note that the MAECHAM/CHEM results are not symmetric about the mean, but have a long tail towards low values.

\section{Model assessments}

In the Arctic the processes leading to stratospheric ozone depletion may undergo too much natural variability to provide 
a definite answer of how ozone will actually evolve. Each model may be considered as supplying a single simulation (or range of simulations in the case of the time slice experiments) of a larger ensemble. The mean of the ensemble can be readily computed, but the atmosphere may in practice evolve in a manner anywhere within, or even outside, the envelope of the model simulations. In the Antarctic, the dominant processes are less dependent on interannual variability and hence the ozone evolution is in principle more predictable. The emphasis here is on the minimum column ozone amount within a region (polar or midlatitudes) as this is of most concern from the point of view of episodes of high surface UV dose. However, minimum ozone can sometimes occur due to the movement of high pressure systems and hence the minimum ozone statistic is not necessarily directly related to chemical depletion. Therefore we also consider briefly the spatially averaged ozone amount.

One of the emphases here is on spring ozone recovery. In view of the range of results obtained, it is important to define this term carefully and it is here used in two senses: (i) the start of ozone recovery, defined as the date of the minimum spring column ozone in the decadally averaged results, (ii) full ozone recovery, defined as the date of the return of the decadally averaged spring column ozone to the value in 1980 .

\subsection{The 1960-2000 time frame: ozone depletion}

As is well established from observations (e.g. WMO, 2002, Chapter 3), polar ozone has been decreasing over this time frame. Figure 9 shows the minimum daily ozone throughout the latitudes $60^{\circ}-90^{\circ} \mathrm{N}$ for the range of models of Table 1 together with TOMS data. For clarity, the models are separated into two panels according to their mode of simulation (transient or time slice). For the transient models, a gaussian filter (full width half maximum of 4.7 years) is fitted through the results for the individual years and the error bars denote two standard deviations from the gaussian mean curve.

For the time slice experiments, the mean and two standard deviations are plotted and the dotted lines give an indication of the temporal behavior of the model results. Each model has a large interannual variability, similar to that of the observations, and hence detecting a signal is difficult. All the models indicate a slight high bias relative to observations. The trends in the local minimum (Table 4) are consistent with the observations, although only MAECHAM/CHEM, E39/C and the observations have statistically significant trends.

Averaging the six model results obtained gives an Arctic trend which is statistically significant and agrees with observations.

In the Antarctic (Fig. 10), the model runs typically agree reasonably well with observations for the past, showing the steady development of the ozone hole during the period. While the interannual variability of most of the models is similar to that observed, both CMAM and UMETRAC have
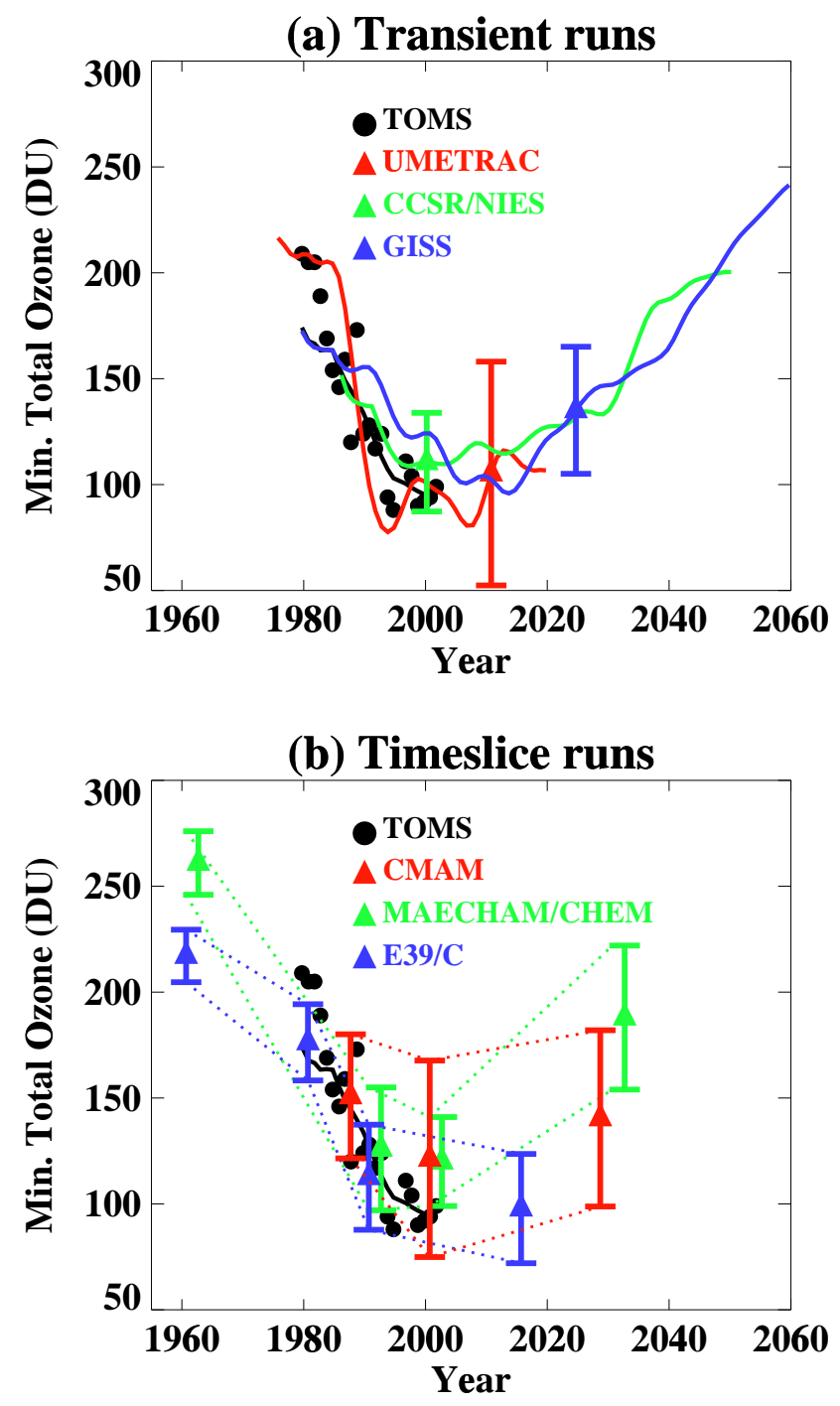

Fig. 10. As in Fig. 9, but for the minimum Antarctic ozone, September to November. For MAECHAM/CHEM only: (i) the values have been plotted two years late for clarity, (ii) a standard tropospheric column of $40 \mathrm{DU}$ has been added to the computed columns above $90 \mathrm{hPa}$.

larger interannual variability, consistent with the large variability seen in the polar temperatures in Fig. 5.

The CCSR/NIES and CMAM results for the past cover a limited range (1986-2000) and this gives rise to a lower trend, as the ozone hole was already well developed by 1986/87. The TOMS results for the same period indicate a trend in the ozone minimum of $-47 \pm 22$, very similar to the CCSR/NIES results. The MAECHAM/CHEM results also have a small trend as the 1979-1999 period could not be matched by the timing of the experiments. The lower variability of most of the models may be an artifact of their cold biases (see Fig. 5), but there is no doubt that CMAM and UMETRAC have more interannual variability than observed. This enhanced variability may be the indirect product of the 
Table 4. Past trends (nominally 1979-1999) in minimum ozone (DU/decade) and in ozone averaged over the polar regions $60-90^{\circ}$ latitude for participating models and TOMS. The error bars are $2 \sigma$ values. The results for the CCSR/NIES and CMAM models cover the periods 19862000 and 1987-2000 respectively; the E39/C and MAECHEM/CHEM results cover the periods 1980-1990 and 1960-1990 respectively

\begin{tabular}{l|c|c|c|c}
\hline Model/Observations & $\begin{array}{c}\text { Arctic trend } \\
\text { Local minimum }\end{array}$ & $\begin{array}{c}\text { Antarctic trend } \\
\text { Local minimum }\end{array}$ & $\begin{array}{c}\text { Arctic trend } \\
\text { Area average, March }\end{array}$ & $\begin{array}{c}\text { Antarctic trend } \\
\text { Area average, October }\end{array}$ \\
\hline UMETRAC Non-orographic gwd & $-6 \pm 22$ & $-80 \pm 31$ & $-17 \pm 20$ & $-60 \pm 24$ \\
CMAM & $-13 \pm 27$ & $-23 \pm 28$ & $-12 \pm 15$ & $-12 \pm 19$ \\
MAECHAM/CHEM & $-16 \pm 10$ & $-45 \pm 3$ & $-10 \pm 3$ & $-32 \pm 2$ \\
E39/C & $-16 \pm 14$ & $-64 \pm 7$ & $-20 \pm 18$ & $-47 \pm 6$ \\
CCSR/NIES & $-33 \pm 38$ & $-41 \pm 21$ & $-23 \pm 55$ & $-26 \pm 30$ \\
GISS & $-21 \pm 33$ & $-34 \pm 12$ & $-20 \pm 18$ & $-44 \pm 8$ \\
\hline Mean (All models) & $-18 \pm 12$ & $-48 \pm 9$ & $-17 \pm 12$ & $-37 \pm 8$ \\
TOMS (Observations) & $-21 \pm 16$ & $-59 \pm 12$ & $-24 \pm 20$ & $-53 \pm 18$ \\
\hline
\end{tabular}

non-orographic gwd schemes in these two models.

The trends averaged over the latitude range $60-90^{\circ}$ are also given in Table 4 and show qualitatively the same features as for the local minima. For the Antarctic, the area-averaged trends for most models are smaller than observed suggesting that models may be underestimating ozone depletion, although this may be partially due to the different time periods considered in the timeslice experiments.

The maximum size of the Antarctic ozone hole during each spring, as given by the area within the $220 \mathrm{DU}$ total ozone contour, is shown in Fig. 11. The results for GISS, E39/C and CCSR/NIES are in good agreement with observations, but may indicate a slight underprediction. A much smaller ozone hole is simulated by UMETRAC, CMAM and MAECHAM/CHEM, related in part to the model high biases in mid-latitudes. Errors in the modelling of the size of the ozone hole can have important implications. Firstly, comparisons between models and observations for ozone amounts near $60^{\circ} \mathrm{S}$ will give poor agreement if the ozone hole is too small, even though the underlying physics of the model may be correct. Secondly, a model with a small ozone hole may evolve differently from the atmosphere due to transport and chemistry effects arising from the radiative perturbation.

\subsection{The 2000-2020 time frame: the start of ozone recovery}

Increases in planetary waves transport more ozone and raise temperatures adiabatically which decreases heterogeneous chemistry, the "dynamical effect on chemistry". Increases in the concentrations of WMGHGs diabatically cool the stratosphere and affect chemistry via reaction rate changes, the "radiative effect on chemistry". If planetary waves increase, the "dynamical" effect increases lower stratospheric ozone and the "radiative" effect decreases ozone, giving a relatively small response. If planetary waves decrease, both the "dynamical" and "radiative" effects are negative, leading to enhanced ozone depletion.

The first signs of ozone recovery are expected within the next two decades (Shindell et al., 1998a; Austin et al., 2000; Schnadt et al., 2002; Rosenfield et al., 2002; Nagashima et al., 2002). Two-dimensional model simulations (e.g. Rosenfield et al., 2002) indicate a slight delay in Arctic and Antarctic spring ozone recovery following the maximum values in halogen loading. The GISS model has a larger response than the other models in the Arctic with the simulation indicating a minimum in the smoothed results of below $175 \mathrm{DU}$ compared with at least 100 DU higher in the other transient runs. The date of the minimum in the decadally averaged Arctic ozone, varies from 2004 for the CCSR/NIES model to 2019 for the GISS model. UMETRAC indicates a minimum at about the year 2015, but the simulation ends shortly afterwards and the results do not change substantially in the final decade. All three transient runs indicate some delay in the onset of ozone recovery, probably due to increases in WMGHGs, although such a result is subject to considerable uncertainty because of the large interannual variability. Although the time slice experiments do not have the temporal resolution to give a precise indication of the timing of future ozone recovery, the E39/C model results (Schnadt et al., 2002) may contrast with the transient model results by suggesting that increases in planetary waves will occur in the Arctic, speeding up ozone recovery. To determine from time slice experiments, whether increases in WMGHGs are delaying the onset of ozone recovery, more simulations are required for the period 1990 to 2015 .

In the Antarctic, the runs are all in fairly good agreement. Of the transient runs, as in the Arctic, the CCSR/NIES model indicates the earliest start of ozone recovery (2001) followed by UMETRAC (2005) and GISS (2008). The minima in the decadally averaged results are all comparable (109, 86 and 98 DU respectively). On the basis of the decadally averaged model results, this would appear to indicate that ozone recovery will begin earlier in the Antarctic than in the Arctic. Such an earlier start to recovery would also be detectable earlier in observations in Antarctica, because of the smaller interannual variability. Observations of the size of the ozone 


\section{Maximum Modelled Antarctic Ozone Hole Area}
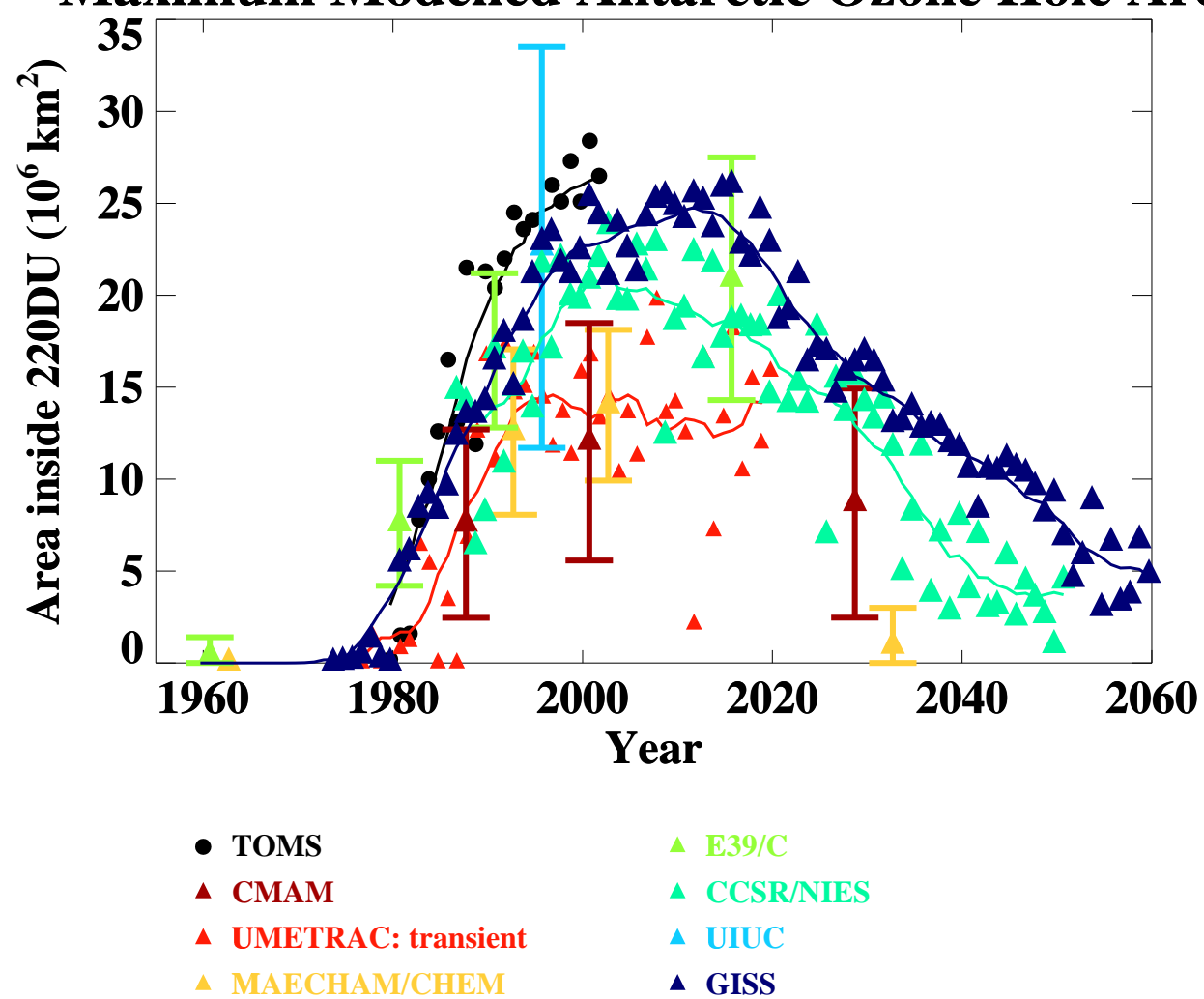

Fig. 11. The maximum area of the ozone hole during the period September to November, as given by the $220 \mathrm{DU}$ contour, for TOMS observations and participating models. The TOMS data and transient model results are plotted for each year and with a gaussian filter applied (solid lines). For the time slice experiments the means and twice the standard deviations of the model samples are included.The GISS results prior to 1974 indicated no ozone values below $220 \mathrm{DU}$ and are not included in the figure.

hole (Fig. 11) do not indicate any clear recovery by October 2001. For the GISS model the maximum size of the ozone hole is in 2015, while for UMETRAC the maximum occurs in 2007 , although any systematic variation is obscured by the large interannual variability noted previously. In contrast the CCSR/NIES results peak as early as the year 2002 .

\subsection{The 2020-2060 time frame: complete ozone recovery}

The return of column ozone to "1980-like conditions", when anthropogenic halogen concentrations were negligible, is here referred to as "complete ozone recovery". As noted in WMO (1999), Chapter 12, this recovery would be to a different vertical distribution of ozone, with higher middle and upper stratospheric ozone. Using a 2-D model, Rosenfield et al. (2002) determined the date for the recovery of total ozone to 1980 levels as a function of day of year and latitude. In the Arctic, this recovery was latest at the end of spring (after 2050) and earliest in autumn (before 2035). Further, the impact of $\mathrm{CO}_{2}$ increases was shown to accelerate the recovery due to increased downwelling, particularly in the Arctic. On the other hand, if $\mathrm{CH}_{4}$ amounts do not increase at the cur- rent rate, ozone recovery could be slowed down in the future by the increased importance of $\mathrm{NO}_{\mathrm{x}}$ chemistry (Randeniya et al., 2002).

The models presented here that have run beyond the year 2020 indicate some recovery in ozone (Figs. 9 and 10). In the Arctic spring, the low values of the GISS model for the decade 2010 to 2020 are no longer present after 2030 while the other models do not show substantial ozone change throughout the period 2020 to 2050. In the Antarctic, the recovery of spring ozone, already underway by 2020, continues in the simulations completed (Fig. 10). The CCSR/NIES and GISS transient model results suggest a near monotonic recovery of ozone.

However, in short duration simulations of UMETRAC spread over several decades the model ozone decreased noticeably over the period 2025 to 2045 (Austin et al., 2001). This was identified as due to increases in ice PSCs as the lower stratospheric climate cools, but would need to be confirmed by model simulations with more detailed PSC schemes. Recovery to 1980-like conditions occurs in the CCSR/NIES and GISS models by about 2045, and perhaps a decade later in the UMETRAC snapshot results (Austin et 
Table 5. As Table 4, but for trends in the minima and means for mid-latitudes, 30-60

\begin{tabular}{l|c|c|c|c}
\hline Model/Observations & $\begin{array}{c}30-60^{\circ} \mathrm{N} \text { trend } \\
\text { Local minimum }\end{array}$ & $\begin{array}{c}30-60^{\circ} \mathrm{S} \text { trend } \\
\text { Local minimum }\end{array}$ & $\begin{array}{c}30-60^{\circ} \mathrm{N} \text { trend } \\
\text { Area average, March }\end{array}$ & $\begin{array}{c}30-60^{\circ} \mathrm{S} \text { trend } \\
\text { Area average, October }\end{array}$ \\
\hline UMETRAC Non-orographic gwd & $-7 \pm 8$ & $-69 \pm 24$ & $-10 \pm 5$ & $-11 \pm 4$ \\
CMAM & $0 \pm 8$ & $-24 \pm 13$ & $-7 \pm 3$ & $-3 \pm 5$ \\
MAECHAM/CHEM & $-3 \pm 2$ & $-14 \pm 3$ & $-6 \pm 1$ & $-7 \pm 1$ \\
E39/C & $-7 \pm 4$ & $-34 \pm 8$ & $-11 \pm 6$ & $-14 \pm 4$ \\
CCSR/NIES & $-4 \pm 7$ & $-58 \pm 35$ & $+2 \pm 13$ & $0 \pm 4$ \\
GISS & $-5 \pm 5$ & $-31 \pm 13$ & $-5 \pm 2$ & $-8 \pm 4$ \\
\hline Mean (All models) & $-4 \pm 3$ & $-38 \pm 9$ & $-6 \pm 3$ & $-7 \pm 2$ \\
TOMS (Observations) & $-10 \pm 10$ & $-45 \pm 14$ & $-14 \pm 10$ & $-10 \pm 7$ \\
\hline
\end{tabular}

al., 2001).

Of the time slice experiments, the MAECHAM/CHEM model indicates a significant long-term Antarctic recovery, consistent with the transient experiments. The CMAM model results for 2028, early in the period, is consistent with MAECHAM/CHEM and with the transient models.

\subsection{Attribution of model polar ozone trends}

In a coupled chemistry-climate model the attribution of ozone trends to dynamical and chemical processes may be ambiguous since the dynamical changes themselves may have been caused by chemical changes to the ozone amounts. Figure 6, illustrating the approximate amounts of PSCs in the model simulations, should in principle reflect the amount of chemical ozone depletion, providing the temporal distributions are not skewed too far towards winter when there may be insufficient sunlight to drive the photochemistry.

This would suggest for example that UMETRAC and CMAM have less Arctic ozone depletion than observed and this is suggested in the Arctic ozone trend (Fig. 9, Table 4) which is smaller than observed, although the difference is not statistically significant. The MAECHAM/CHEM, E39/C and CCSR/NIES results for PSCs in Fig. 6 suggest that their chemical ozone depletion is larger than observed in the Arctic, although the net ozone trend is similar to observations. In the latter two models this is resolved by the use of a "nucleation barrier" which results in fewer PSCs more consistent with the large scale observed temperature fields.

When coupled with the halogen loading, the areas indicated in Fig. 6 should be a first order indication of PSC impact on chemistry with the above caveat on the use of a nucleation barrier in some models. The fact that a model such as UMETRAC with non-orographic gwd can reproduce to first order the observed areas near PSC threshold temperatures as well as the observed ozone trend indicates that a nucleation barrier is not necessary. Hence, although the use of a nucleation barrier may be justified on the basis of the physics (see Sect. 2.1/E39/C) a stronger argument may be that small scale processes unrepresented by the model large scale fields provides the necessary nucleation barrier (see Sect. 2.1/ECHAM/CHEM). Clearly, the first priority of a model is to produce physically realistic results which are only achievable given small temperature biases. The CMAM model result for 2028, early in this period, is consistent with MAECHAM/CHEM and with the transient models.

\subsection{Ozone changes in mid-latitudes}

The minimum spring ozone columns in mid-latitudes are shown in Fig. 12 and the trends computed are given in Table 5. The results for minimum ozone are similar to the results in polar latitudes, discussed in Sects. 4.1-4.3. In northern mid-latitudes most of the models are almost constant throughout the integration but in the timeslice experiments there is a just perceptible minimum near the year 2000. In UMETRAC, the decadally smoothed results decrease steadily until the end of the integration (2020). Most of the model results indicate a statistically significant past decrease in area-averaged ozone and although the results are consistent with observations, the trends are rather small with large error bars. The UMETRAC model results have a high bias relative to observations, primarily due to excessive ozone in the lower stratosphere caused by insufficient local $\mathrm{NO}_{\mathrm{x}}$ amounts. MAECHAM/CHEM total ozone in midlatitudes is high because stratosphere-troposphere exchange is overestimated, a numerical problem largest in midlatitude spring. The absolute changes with time are, however, consistent with the other models.

In southern mid-latitudes, the area averaged ozone amounts, both in the models and in the TOMS data, are very similar to the area averaged results for northern midlatitudes. A different picture occurs, however, when investigating local minima (Fig. 12, lower panel), which reflects the development and decay of the Antarctic ozone hole. In the decadally averaged results, the lowest values occur in 1999 (UMETRAC) and 2002 (CCSR/NIES) but the decadal averages do not change appreciably from about the year 1995 onwards and hence the date of the start of ozone recovery is very dependent on the method used to diagnose that recovery. 

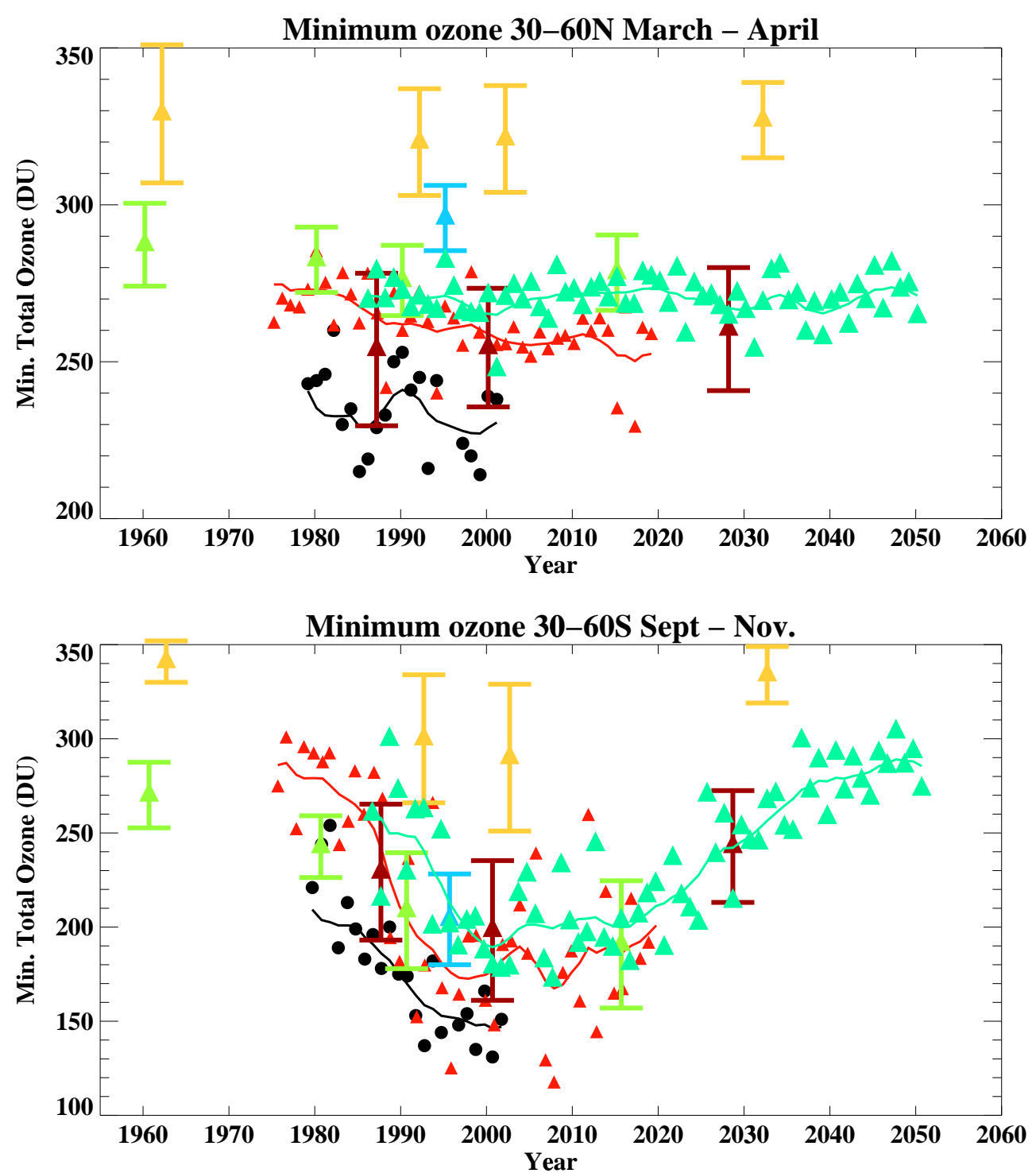

Fig. 12. Minimum total column ozone in the latitude range $30^{\circ}$ to $60^{\circ}$ in the Northern (upper) and Southern (lower) Hemispheres for participating models. See Fig. 11 for line styles and symbols.

\section{Conclusions}

In this paper the results from a number of 3-D chemistryclimate models have been compared amongst themselves and with observations, covering a range of diagnostics for processes influencing ozone, with a particular emphasis on the polar regions. The work brings together models, mostly with detailed stratospheric chemistry schemes, with a range of spatial resolution. In addition, the models have been run in two different modes: time slice and transient. In the former, the greenhouse gas concentrations and halogen amounts are fixed at concentrations specific to a given year; in the latter, the concentrations evolve in accordance with observations and future anticipated values. By comparing the model results an estimate of the future evolution of ozone is obtained, together with an indication of the likely uncertainties.

The main uncertainties of 3-D coupled chemistry-climate models stem from the performance of the underlying dynamical model. Cold biases have been found to exist in the stratosphere of many of the models, consistent with that previously found for models without chemistry (Pawson et al., 2000). The temperature is correlated with the lower stratospheric heat flux, which is a measure of wave propagation from the troposphere. Consequently, temperature bias comes from insufficient wave propagation or a failure to represent the heat flux-temperature relationship, or both (Newman et al., 2001). The results here suggest that the incorporation of non-orographic gravity wave drag can reduce the temperature bias, particularly in the southern hemisphere, consistent 
with Manzini and McFarlane (1998), while increased horizontal resolution can improve the heat flux-temperature relationship in the northern hemisphere. In the lower stratosphere, most models have a cold bias in the Antarctic spring, but a warm bias in the Arctic in late winter. The impact of the cold biases would be to simulate larger amounts of PSCs than are observed. Some models introduce a nucleation barrier, which helps to reduce the impact of the temperature bias on ozone, while other models do not, assuming that small scale processes achieve the desired low temperatures sufficient to trigger nucleation. In consequence, at the current stage of model development, uncertainties in the details of PSC formation and sedimentation are probably less important than the model temperature biases in simulating accurate ozone amounts. However, the timing of the temperature biases can change the seasonal evolution of ozone depletion relative to that observed, and affect the conclusions regarding the timing of ozone recovery.

Model chemical transport influences the performance of coupled chemistry climate models. At least for the current atmosphere, modelled chemical transport in the lower stratosphere is not sensitive to the position of the upper boundary, providing sufficient vertical resolution is present up to the middle stratosphere. However, low top models need higher dissipation immediately below their top than the equivalent higher top models, and this additional dissipation may lead to a reduced response to climate change. Further, low top models are unable to accommodate non-orographic gwd schemes and partly artificial constructs, such as a nucleation barrier for PSC formation, may need to be included to compensate for the cold bias. There is a tendency for the models described here to underestimate transport in winter, because of the strong vortex, and overestimate transport in summer. This issue can again be addressed with a non-orographic gwd scheme. Secondly, a major issue for future Arctic ozone amounts is whether wave propagation will increase or decrease in the future. If wave propagation decreases, less ozone is transported into the polar regions and by the noted relationship between heat flux and temperature, the lower stratosphere cools and ozone depletion increases. The opposite occurs for an increase in wave propagation. Models are qualitatively consistent with observations in showing a decreasing trend in wave propagation for the past. However, the observed trend is only just statistically significant and the model results presented here have trends which are lower than observed by at least a factor of two and are typically not statistically significant. Future predictions are much more uncertain, with most models showing a continuing downward trend into the future, but at least one other model indicating an increase.

The behaviour of the long-lived species could be equally important in controlling ozone. Water vapour concentrations have been increasing in the statosphere for several decades (Oltmans et al., 2000; Rosenlof et al., 2001). This would have had a significant cooling effect (Forster and Shine,
1999; Oinas et al., 2001) resulting in enhanced PSC formation and increased ozone loss (Kirk-Davidoff et al., 1999). However, in climate models, the water vapour trend tends to be driven by the cold trap mechanism and methane oxidation, which together contribute less than half the observed trend in the stratosphere (e.g. Austin, 2002; Schnadt et al., 2002). Increased water vapour also affects homogeneous ozone chemistry via the $\mathrm{OH}$ radical, reducing ozone in the upper and lower stratosphere and delaying mid-latitude ozone recovery by 10 to 20 years (e.g. Dvortsov and Solomon, 2001; Shindell, 2001). Another uncertainty is the presence of aerosol due to future unpredictable volcanic eruptions. For a large eruption such as that of Mt. Pinatubo, in addition to the radiative perturbation (e.g. Stenchikov et al., 1998) sufficient aerosol would be present to provide additional sites for heterogeneous chemistry and possibly severe ozone loss for a period of a few years (e.g. Solomon et al., 1996).

The results of the 3-D coupled chemistry-climate model simulations presented here now provide a better indication of likely future changes than was previously possible. For the transient model simulations the start of ozone recovery, defined as the date when the decadally averaged minimum ozone first starts to increase, occurs in the Antarctic in the range 2001 to 2008, depending on the model, and in the Arctic occurs in the range 2004 to 2019. In the Antarctic, however, the vertical and horizontal extent of the ozone hole may increase slightly further over the next few years. Thus, the results here suggest that the start of ozone recovery will occur slightly later in the Arctic than in the Antarctic and that the start of ozone recovery in the Arctic may be delayed by a few years by greenhouse gas increases. While the statistical basis of this result is not high, a plausible explanation would be that in the Antarctic, the depth of the ozone hole (but not its area) depend strongly on halogen loading, with climate change a minor contributor. In the Arctic, it is the cold winters, which are affected by climate change, that determine more the ozone depletion rather than the amount of halogen loading. Trends in Arctic minimum ozone are highly dependent on the period over which the trend is computed because of large interannual variability and the gradual reduction of ozone depletion rates in recent years. Although in some cases the trend in the area-averaged ozone has higher statistical significance and is more robust to changes in averaging period, the work here has focussed on ozone minima because of its relationship with extreme UV episodes. Both statistics give qualitatively similar results in terms of the timing of ozone recovery. The results for local minima in mid-latitudes indicate a similar timing for ozone recovery, probably due to the high importance of transport from high latitudes. The past trends in the area-averaged results for mid-latitudes are similar in the northern and southern hemispheres, both in the models and in the observations. Interannual variability on the sub-decadal time scale may still lead to ozone extremes. In the worst case scenario, therefore, it may take until at least the end of the 2020's before we can be certain that ozone re- 
covery has started in the Arctic or in northern mid-latitudes. To put this into perspective, most models predict relatively modest changes in future spring Arctic column ozone (under $10 \%$ ). These long time scales are consistent with previous estimates (Weatherhead et al., 2000) of the time required to detect small ozone trends.

Towards the middle of the 21st century, model predictions appear to be more uncertain. Hence, although recovery of ozone to 1980-like conditions is to be expected by about 2050 (e.g. Rosenfield et al., 2001), models will need to have a better representation of the water vapour increase than has hitherto been possible for full confidence in their predictions. In this work, the few models which have been integrated to this time also indicate a full ozone recovery in the Antarctic by about 2050. Definitive information for the Arctic is even less certain, but because of the relatively modest change in ozone indicated, it is possible that "full recovery" may occur somewhat earlier there.

Acknowledgements. JA was supported by the European Community, under the EuroSPICE project and by the UK Government Meteorological Research Programme. Climate modeling at GISS is supported by NASA's Atmospheric Chemistry Modeling and Analysis Program. CMAM research is supported by the Natural Sciences and Engineering Research Council, the Meteorological Service of Canada, and the Canadian Foundation for Climate and Atmospheric Sciences. The MAECHAM/CHEM simulations were carried out within the KODYACS and MEDEC projects (AFO 2000 programme), funded by the German government. The ERA streamfunction analyses were kindly supplied by Christine Land (MPI Hamburg). Development and application of UIUC model were supported by the U.S. National Science Foundation (NSF) and the Carbon Dioxide Research Program, Environmental Sciences Division of the U.S. Department of Energy under grant ATM 95-22681, and by the NSF under grant ATM 00-0084270. Björn Knudsen and two anonymous referees are thanked for their helpful comments on the text.

\section{References}

Austin, J.: A three-dimensional coupled chemistry-climate model simulation of past stratospheric trends, J. Atmos. Sci., 59, 218232, 2002.

Austin, J. and Butchart, N.: Coupled chemistry-climate model simulations for the period 1980 to 2020: ozone depletion and the start of ozone recovery, Q. J. R. Meteorol. Soc., submitted, 2002.

Austin, J., Butchart, N., and Shine, K. P.: Possibility of an Arctic ozone hole in a doubled- $\mathrm{CO}_{2}$ climate, Nature, 360, 221-225, 1992.

Austin, J., Butchart, N., and Swinbank, R. S.: Sensitivity of ozone and temperature to vertical resolution in a GCM with coupled stratospheric chemistry, Q. J. R. Meteorol. Soc., 123, 1405-1431, 1997.

Austin, J., Knight, J., and Butchart, N.: Three-dimensional chemical model simulations of the ozone layer: 1979-2015, Q. J.R. Meteorol. Soc., 126, 1533-1556, 2000.
Austin, J., Butchart, N., and Knight, J.: Three-dimensional chemical model simulations of the ozone layer: 2015-2055, Q. J.R. Meteorol. Soc., 127, 959-974, 2001.

Baldwin, M. P. and Dunkerton, T. J.: Propagation of the Arctic Oscillation from the stratosphere to the troposphere, J. Geophys. Res., 104, 30 937-30 946, 1999.

Baldwin, M. P. and Dunkerton, T. J.: Stratospheric harbingers of anomalous weather regimes, Science, 294, 581-584, 2001.

Beagley, S. R., de Grandpré, J., Koshyk, J. N., McFarlane, N. A., and Shepherd, T. G.: Radiative-dynamical climatology of the first-generation Canadian Middle Atmosphere Model, Atmos.Ocean, 35, 293-331, 1997.

Beagley, S. R., McLandress, C., Fomichev, V. I., and Ward, W. E.: The extended Canadian middle atmosphere model, Geophys. Res. Lett., 27, 2529-2532, 2000.

Boer, G. J., Flato, G., and Ramsden, D.: A transient climate change simulation with greenhouse gas and aerosol forcing: projected climate to the twenty-first century, Clim. Dyn., 16, 427-450, 2000.

Brühl, C., Steil, B., and Manzini, E.: Feedback processes between chemistry and meteorology with focus on lower stratospheric polar vortices, simulations with a coupled GCM, Proceedings SPARC, November 2000, Mar del Plata, Argentina, Available at the SPARC website or CD-ROM), 2001.

Butchart, N. and Austin, J.: Middle atmosphere climatologies from the troposphere-stratosphere configuration of the UKMO's Unified Model, J. Atmos. Sci., 55, 2782-2809, 1998.

Carslaw, K., Oelhaf, H., Crowley, J., Goutail, F., Knudsen, B., Larsen, N., Redaelli, G., Rex, M., Roscoe, H., Ruhnke R., and Volk, M.: Polar Ozone, European Research in the Stratosphere 1996-2000, Advances in our understanding of the ozone layer during THESEO, Directorate-General for Research, Environment and sustainable development programme, Office for Official Publications of the European Communities EUR 19867, Chapter 3, 69-132, 2001.

Dameris, M., Grewe, V., Hein, R., and Schnadt, C.: Assessment of future development of the ozone layer, Geophys. Res. Lett., 25, 3579-3582, 1998.

de Grandpré, J., Beagley, S. R., Fomichev, V. I., Griffioen, E., McConnell, J. C., Medvedev, A. S., and Shepherd, T. G.: Ozone climatology using interactive chemistry: results from the Canadian Middle Atmosphere Model, J. Geophys. Res., 105, 26475 26 491, 2000.

de Grandpré, J., Sandilands, J. W., McConnell, J. C., Beagley, S. R., Croteau, P. C., and Danilin, M. Y.: Canadian Middle Atmosphere Model: Preliminary results from the chemical transport module, Atmos.-Ocean, 35, 385-431, 1997.

DeMore, W. B., Sander, S. P., Golden, D. M., Hampson, R. F., Kurylo, M. J., Howard, C. J., Ravishankara, A. R., Kolb, C. E., and Molina, M. J.: Chemical kinetics and photochemical data for use in stratospheric modeling, Evaluation number 12, JPL Publication 97-4, Pasadena, Ca, 1997.

Dvortsov, V. L. and Solomon, S.: Response of the stratospheric temperatures and ozone to past and future increases in stratospheric humidity, J. Geophys. Res., 106, 7505-7514, 2001.

Dye, J., Baumgardner, D., Gandrud, B. W., Kawa, S. R., Kelly, K. K., Loewenstein, M., Ferry, G. V., Chan, K. R., and Gary, B. L.: Particle size distributions in Arctic polar stratospheric clouds, growth and freezing of sulfuric acid droplets and impli- 
cations for cloud formation, J. Geophys. Res., 97, 8015-8034, 1992.

Edmon, H. J., Hoskins, B. J., and McIntyre, M. E.: Eliassen-Palm cross-sections for the troposphere, J. Atmos. Sci., 37, 26002616, 1980. Also corrigendum, J. Atmos. Sci., 38, 1115, 1980.

Egorova, T. A., Rozanov, E. V., Schlesinger, M. E., Andronova, N. G., Malyshev, S. L., Karol, I. L., and Zubov, V. A.: Assessment of the effects of the Montreal protocol on atmospheric ozone, Geophys. Res. Lett., 28, 2389-2392, 2001.

Fahey, D. W., Gao, R. S., Carslaw, K. S., et al.: The detection of large $\mathrm{HNO}_{3}$-containing particles in the winter arctic stratosphere, Science, 291, 1026-1031, 2001.

Farman, J. C., Gardner, B. G., and Shanklin, J. D.: Large losses of total ozone in Antarctica reveal seasonal $\mathrm{ClO}_{\mathrm{x}} / \mathrm{NO}_{\mathrm{x}}$ interaction, Nature, 315, 207-210, 1985.

Forster, P. M. de F. and Shine, K. P.: Stratospheric water vapor changes as a possible contributor to observed stratospheric cooling, Geophys. Res. Lett., 26, 3309-3312, 1999.

Garcia, R. R. and Boville, B. A.: "Downward control" of the mean meridional circulation and temperature distribution of the polar winter stratosphere, J. Atmos. Sci., 51, 2238-2245, 1994.

Gibson, J. L., Kallberg, P., Uppala, S., Hernandez, A., Nomura, A., and Serrano, E.: ERA description. ECMWF Reanalysis Report, 1, pp. 72, 1997.

Gillett, N. P., Allen, M. R. and Williams, K. D.: The role of stratospheric resolution in simulating the Arctic Oscillation response to greenhouse gases, Geophys. Res. Lett., 29, 10.1029/2001GL014444, 2002.

Gleckler, P. E.: AMIP Newsletter: AMIP-II guidelines, Lawrence Livermore National Laboratory, Livermore, CA, 1996.

Groves, K. S. and Tuck, A. F.: Stratospheric $\mathrm{O}_{3}-\mathrm{CO}_{2}$ coupling in a photochemical model. I: Without chlorine chemistry, II: With chlorine chemistry, Q. J. R. Meteorol. Soc., 106, 125-157, 1980.

Hanson, D. and Mauersberger, K.: Laboratory studies of the nitric acid trihydrate: Implications for the south polar stratosphere, Geophys. Res. Lett., 15, 855-858, 1988.

Hartmann, D. L., Holton, J. R., and Fu, Q.: The heat balance of the tropical tropopause, cirrus, and stratospheric dehydration, Geophys. Res. Lett., 28, 1969-1972, 2001.

Haynes, P. H., Marks, C. J., McIntyre, M. E., Shepherd, T. G., and Shine, K. P.: On the "downward control" of extratropical diabatic circulations by eddy-induced mean zonal forces, J. Atmos. Sci., 48, 651-678, 1991

Hein, R., Dameris, M., Schnadt, C., Land, C., Grewe, V., Kohler, I., Ponater, M., Sausen, R., Steil, B., Landgraf, J., and Brühl, C.: Results of an interactively coupled atmospheric chemistry general circulation model: comparison with observations, Ann. Geophysicae, 19, 435-457, 2001.

Hines, C. O.: Doppler spread parameterization of gravity wave momentum deposition in the middle atmosphere, Part 1: Basic formulation, Part 2: Broad and quasi-monochromatic spectra and implementation, J. Atmos. Solar Terr. Phys., 59, 371-400, 1997.

Hood, L., Rossi, S., and Beulen, M.: Trends in lower stratospheric zonal winds, Rossby wave breaking behavior, and column ozone at northern midlatitudes, J. Geophys. Res., 104, 24 321-24339, 1999.

IPCC: Intergovernmental Panel on Climate Change, Climate change: the supplementary report to the IPCC scientific assessment, (Eds.) Houghton, J. T., Callander, B. A., and Varney, S. K.,
Cambridge University Press, Cambridge, UK, 1992.

IPCC: Climate Change 2001, The Scientific Basis, Contribution of Working Group I to the Third Assessment Report of the Ingovernmental Panel on Climate Change, (Eds.) Houghton, J. T., Ding, Y., Griggs, D. J., Noguer, M., van der Linden, P. J., Dai, X., Maskell, K., and Johnson, C. A., Cambridge University Press, 2001.

Kirk-Davidoff, D. B., Hintsa, E. J., Anderson, J. G., and Keith, D. W.: The effect of climate change on ozone depletion through changes in stratospheric water vapour, Nature, 402, 399-401, 1999.

Kodera, K. and Koide, H.: Spatial and seasonal characteristics of recent decadal trends in the Northern Hemisphere troposphere and stratosphere, J. Geophys. Res., 102, 19433-19447, 1997.

Kodera, K., Chiba, M., Koide, H., Kitoh, A., and Nikaidou, Y. Interannual variability of the winter stratosphere and troposphere in the Northern Hemisphere, J. Meteorol. Soc. Japan., 74, 365$382,1996$.

Kuroda, Y. and Kodera, K.: Role of planetary waves in the stratosphere-troposphere coupled variability in the Northern Hemisphere winter, Geophys. Res. Lett., 26, 2375-2378, 1999.

Land, C., Ponater, M., Sausen, R., and Roeckner, E.: The ECHAM4.L39(DLR) atmosphere GCM - Technical description and model climatology, Report No. 1991-31, DLR Oberpfaffenhofen, Weßling, Germany, ISSN 1434-8454, 1999.

Landgraf, J. and Crutzen, P. J.: An efficient method for online calculations of photolysis and heating rates, J. Atmos. Sci., 55, 863 878, 1998

Lawrence, B. N.: Some aspects of the sensitivity of stratospheric climate simulation to model lid height, J. Geophys. Res., 102, 23 805-23 811, 1997.

McFarlane, N. A.: The effect of orographically excited gravity wave drag on the general circulation of the lower stratosphere and troposphere, J. Atmos. Sci., 44, 1775-1800, 1987.

McLandress, C.: On the importance of gravity waves in the middle atmosphere and their parameterization in general circulation models, J. Atmos. Sol.-Terr. Phys., 60, 1357-1383, 1998.

McLandress, C.: Interannual variations of the diurnal tide in the mesosphere induced by a zonal-mean wind oscillation in the tropics, Geophys. Res. Lett., 29, 10.1029/2001GL014551, 2002.

Manzini, E. and McFarlane, N. A.: The effect of varying the source spectrum of a gravity wave parameterization in a middle atmosphere general circulation model, J. Geophys. Res., 103, 31 523$31539,1998$.

Manzini, E., McFarlane, N. A., and McLandress, C.: Impact of the Doppler Spread Parameterization on the simulation of the middle atmosphere circulation using the MA/ECHAM4 general circulation model, J. Geophys. Res., 102, 25 751-25 762, 1997.

Manzini, E., Steil, B., Brühl, C., Giorgetta, M., and Krüger, K.: A new interactive chemistry climate model. 2: Sensitivity of the middle atmosphere to ozone depletion and increase in greenhouse gases: implications for recent stratospheric cooling, J. Geophys. Res., submitted, 2002.

Medvedev, A. S., Klaassen, G. P., and Beagley, S. R.: On the role of anisotropic gravity wave spectrum in maintaining the circulation of the middle atmosphere, Geophys. Res. Lett., 25, 509-512, 1998.

Medvedev, A. S. and Klaassen, G. P.: Vertical evolution of gravity wave spectra and the parameterization of associated wave drag, 
J. Geophys. Res., 100, 25 841-25 853, 1995.

Miller, M. J., Palmer, T. N., and Swinbank, R.: Parameterization and influence of sub-grid scale orography in general circulation and numerical weather prediction models, Meteorol. Atmos. Phys., 40, 84-109, 1989.

Murray, F. W.: On the computation of saturation vapor pressure, J. Appl. Meteorol., 6, 203-204, 1967.

Nagashima, T., Takahashi, M., Takigawa, M., and Akiyoshi, H.: Future development of the ozone layer calculated by a general circulation model with fully interactive chemistry, Geophys. Res. Lett., 10.1029/2001GL014026, 2002.

Newman, P. A. and Nash, E. R.: Quantifying the wave driving of the stratosphere, J. Geophys. Res., 105, 12 485-12 497, 2000.

Newman, P. A., Nash, E. R., and Rosenfield, J. E.: What controls temperature of the Arctic stratosphere during spring?, J. Geophys. Res., 106, 19999-20010, 2001.

Ohhashi, Y. and Yamazaki, K.: Variability of the Eurasian pattern and its interpretation by wave activity flux, J. Meteorol. Soc. Japan, 77, 495-511, 1999.

Oinas, V., Lacis, A. A., Rind, D., Shindell, D. T., and Hansen, J. E.: Radiative cooling by stratospheric water vapor: big differences in GCM results, Geophys. Res. Lett., 28, 2791-2794, 2001.

Oltmans, S. J., Vomel, H., Hofmann, D. J., Rosenlof, K. H., and Kley, D.: The increase in stratospheric water vapor from balloonborne, frostpoint hygrometer measurements at Washington, D. C., and Boulder, Colorado, Geophys. Res. Lett., 27, 3453-3456, 2000.

Palmer, T. N., Shutts, G. J., and Swinbank, R.: Alleviation of a systematically westerly bias in general circulation and numerical weather prediction models through an orographic gravity wave drag parametrization, Q. J. R. Meteorol. Soc., 112, 1001-1039, 1986.

Pawson, S., Krüger, K., Swinbank, R., Bailey, M., and O’Neill, A.: Intercomparison of two stratospheric analyses: temperatures relevant to polar stratospheric cloud formation, J. Geophys. Res., 104, 2041-2050, 1999.

Pawson, S. and Naujokat, B.: Trends in daily wintertime temperatures in the northern stratosphere, Geophys. Res. Lett., 24, 575578, 1997.

Pawson, S., Kodera, K., Hamilton, K., et al.: The GCM-reality intercomparison project for SPARC (GRIPS): Scientific issues and initial results, Bull. Amer. Met. Soc., 81, 781-796, 2000.

Perlwitz, J., Graf, H.-F., and Voss, R.: The leading variability mode of the coupled troposphere-stratosphere winter circulation in different climate regimes, J. Geophys. Res., 105, 6915-6926, 2000.

Peter, T., Brühl, C., and Crutzen, P. J.: Increase of the PSCformation probability caused by high-flying aircraft, Geophys. Res. Lett., 18, 1465-1468, 1991.

Pitari, G., Palermi, S., Visconti, G., and Prinn, R. G.: Ozone response to a $\mathrm{CO}_{2}$ doubling: Results from a stratospheric circulation model with heterogeneous chemistry, J. Geophys. Res., 97, 5953-5962, 1992.

Pitari, G., Mancini, E., Rizi, V., and Shindell, D.: Impact of future climate and emission changes on stratospheric aerosols and ozone, J. Atmos. Sci., 59, 414-440, 2002.

Pullen, S. and Jones, R. L.: Accuracy of temperatures from UKMO analyses of 1994/95 in the Arctic winter stratosphere, Geophys. Res. Lett., 24, 845-848, 1997.

Randel, W. J., Wu, F., and Stolarski, R.: Changes in column ozone correlated with the stratospheric EP flux, J. Meteor. Soc. Japan, 80, 849-862, 2002.

Randeniya, L. K., Vohralik, P. F., and Plumb, I. C.: Stratospheric ozone depletion at northern midlatitudes in the $21 \mathrm{st}$ century: the importance of future concentrations of greenhouse gases nitrous oxide and methane, Geophys. Res. Lett, 29, 10.1029/2001GL014295, 2002.

Rasch, P. J. and Lawrence, M.: Recent development in transport methods at NCAR, MPI-Report No. 265, Max-Planck-Institut für Meteorologie, FRG, pp. 65-75, 1998.

Rind, D., Suozzo, R., Balachandran, N. K., Lacis, A., and Russell, G.: The GISS global climate/middle atmosphere model, I, Model structure and climatology, J. Atmos. Sci., 45, 329-370, 1988a.

Rind, D., Suozzo, R., and Balachandran, N. K.: The GISS global climate/middle atmosphere model, II, Model variability due to interactions between planetary waves, the mean circulation and gravity wave drag, J. Atmos. Sci., 45, 371-386, 1988 b.

Rind, D., Shindell, D., Lonergan, P., and Balachandran, N. K.: Climate change and the middle atmosphere: Part III: The doubled CO2 climate revisited, J. Climate, 11, 876-894, 1998.

Roeckner, E., Bengtsson, L., Feichter, J., Lelieveld, J., and Rodhe, H.: Transient climate change simulations with a coupled atmosphere-ocean GCM including the tropospheric sulfur cycle, J. Climate, 12, 3003-3032, 1999.

Rosenfield, J. E., Douglass, A. R., and Considine, D. B.: The impact of increasing carbon dioxide on ozone recovery, J. Geophys. Res., 10.1029/2001JD000824, 2002.

Rosenlof, K. H., Oltmanns, S. J., Kley, D., et al.: Stratospheric water vapor increases over the past half-century, Geophys. Res. Lett., 28, 1195-1198, 2001.

Rozanov, E. V., Zubov, V. A., Schlesinger, M. E., Yang, F., and Andronova, N. G.: The UIUC 3-D Stratospheric Chemical Transport Model: Description and Evaluation of the Simulated Source Gases and Ozone, J. Geophys. Res., 104, 11 755-11 781, 1999.

Rozanov, E. V., Schlesinger, M. E., and Zubov, V. A.: The University of Illinois at Urbana-Champaign three-dimensional stratosphere-troposphere general circulation model with interactive ozone photochemistry: Fifteen-year control run climatology, J. Geophys. Res., 106, 27 233-27 254, 2001.

Sander, S. P., Ravishankara, A. R., Friedl, R. R., Demore, W. B., Golden, D. M., Kolb, C. E., Kurylo, M. J., Molina, M. J., Hampson, R. F., Huie, R. E., and Moortgat, G. K.: Chemical kinetics and photochemical data for use in stratospheric modeling, Evaluation number 12: Update of key reactions, JPL Publication 00-3, Pasadena, Ca., 2000.

Scaife, A. A., Butchart, N., Warner, C. D., Stainforth, D., Norton, W., and Austin, J.: Realistic quasi-biennial oscillations in a simulation of the global climate, Geophys. Res. Lett., 27, 3481-3484, 2000.

Schlager, H., Arnold, F., Hofmann, D. J., and Deshler, T.: Balloon observations of nitric acid aerosol formation in the arctic stratosphere: I Gaseous nitric acid, Geophys. Res. Lett., 17, 12751278, 1990.

Schnadt, C., Dameris, M., Ponater, M., Hein, R., Grewe, V., and Steil, B.: Interaction of atmospheric chemistry and climate and its impact on stratospheric ozone, Clim. Dyn., 18, 501-517, 2002.

Shea, D. J., Trenberth, K. E., and Reynolds, R. W.: A global monthly sea surface temperature climatology, NCAR Technical 
Note NCAR/TN345+STR, pp. 167, 1990.

Shepherd, T. G., Semeniuk, K., and Koshyk, J.: Sponge-layer feedbacks in middle atmosphere models, J. Geophys. Res., 101, 23 447-23 464, 1996.

Shindell, D. T.: Climate and ozone response to increased stratospheric water vapor, Geophys. Res. Lett., 28, 1551-1554, 2001.

Shindell, D. T., Rind, D., and Lonergan, P.: Increased polar stratospheric ozone losses and delayed eventual recovery owing to increasing greenhouse-gas concentrations, Nature, 392, 589-592, 1998a.

Shindell, D. T., Rind, D., and Lonergan, P.: Climate change and the middle atmosphere, IV, Ozone photochemical response to doubled $\mathrm{CO}_{2}$, J. Clim., 11, 895-918, 1998 b.

Shindell, D. T., Schmidt, G. A., Miller, R. L., and Rind, D.: Northern Hemisphere winter climate response to greenhouse gas, volcanic, ozone and solar forcing, J. Geophys. Res., 106, 71937210, 2001.

Solomon, S., Portmann, R. W., Garcia, R. R., Thomason, L. W., Poole, L. R., and McCormick, M. P.: The role of aerosol variations in anthropogenic ozone depletion at northern midlatitudes, J. Geophys. Res., 101, 6713-6727, 1996.

Solomon, S.: On the depletion of Antarctic ozone, Nature, 321, 755-758, 1986.

Steil, B., Brühl, C., Manzini, E., Crutzen, P. J., Lelieveld, J., Rasch, P. J., Roeckner, E., and Krüger, K.: A new interactive chemistry climate model. 1: Present day climatology and interannual variability of the middle atmosphere using the model and 9 years of HALOE/UARS data, J. Geophys. Res., Submitted, 2002.

Steil, B., Dameris, M., Brühl, C., Crutzen, P. J., Grewe, V., Ponater, M., and Sausen, R.: Development of a chemistry module for GCMs: first results of a multi-year integration, Ann. Geophysicae, 16, 205-228, 1998.

Stenchikov, G. L., Kirchner, I., Robock, A., Graf, H.-F., Antuña, J. C., Grainger, R. G., Lambert, A., and Thomason, L.: Radiative forcing from the 1991 Mount Pinatubo volcanic eruption, J. Geophys. Res., 103, 13 837-13 857, 1998.

Swinbank, R. and O'Neill, A.: A stratosphere-troposphere data assimilation system, Mon. Weather Rev., 122, 686-702, 1994.

Takigawa, M., Takahashi, M., and Akiyoshi, H.: Simulation of ozone and other chemical species using a Center for Climate Systems Research/National Institute for Environmental Studies atmospheric GCM with coupled stratospheric chemistry, J. Geo- phys. Res., 104, 14 003-14 018, 1999.

Tanaka, H. L., Kanohgi, R., and Yasunari, T.: Recent abrupt intensification of the northern polar vortex since 1988, J. Meteorol. Soc. Japan, 74, 947-954, 1996.

Timmreck, C. and Graf, H.-F.: A microphysical model for simulation of stratospheric aerosol in a climate model, Meteorol. Zeitschrift, 9, 263-282, 2000.

Waibel, A. E., Peter, T., Carslaw, K. S., Oelhaf, H., Wetzel, G., Crutzen, P. J., Pöschl, U., Tsias, A., Reimer, E., and Fischer, H.: Arctic ozone loss due to denitrification, Science, 283, 2064 2069, 1999.

Warner, C. D. and McIntyre, M. E.: Toward an ultra-simple spectral gravity wave parameterization for general circulation models, Earth Planets Space, 51, 475-484, 1999.

Waugh, D. W., Randel, W. J., Pawson, S., Newman, P. A., and Nash, E. R.: Persistence of the lower stratospheric polar vortices, J. Geophys. Res., 104, 27 191-27 201, 1999.

Weatherhead, E. C., Reinsel, G. C., Tiao, G. C., Jackman, C. H., Bishop, L., Hollandsworth Frith, S. M., DeLuisi, J., Keller, T., Oltmans, S. J., Fleming, E. L., Wuebbles, D. J., Kerr, J. B., Miller, A. J., Herman, J., McPeters, R., Nagatani, R. M. and Frederick, J. E.: Detecting the recovery of total column ozone. J. Geophys. Res., 105, 22 201-22 210, 2000.

WMO: (World Meteorological Organization), Scientific assessment of ozone depletion: 1991, WMO Gobal Ozone Research and Monitoring Project, Report No. 25, Geneva, Switzerland, 1992.

WMO: Scientific Assessment of Ozone depletion: 1998, WMO Gobal Ozone Research and Monitoring Project, Report No. 44, Geneva, Switzerland, 1999.

WMO: Scientific Assessment of Ozone depletion: 2002, WMO Gobal Ozone Research and Monitoring Project, Geneva, Switzerland, in press, 2002.

Yang, F., Schlesinger, M. E., and Rozanov, E.: Description and performance of the UIUC 24-layer stratosphere/troposphere general circulation model, J. Geophys. Res., 105, 17 925-17 954, 2000.

Zubov, V. A., Rozanov, E. V., and Schlesinger, M. E.: Hybrid scheme for three-dimensional advective transport, Mon. Wea. Rev., 127, 1335-1346, 1999.

Zurek, R. W., Manney, G. L., Miller, A. J., Gelman, M. E., and Nagatani, R. M.: Interannual variability of the north polar vortex in the lower stratosphere during the UARS mission, Geophys. Res. Lett., 23, 289-292, 1996. 